\title{
Multifrequency behavior of classical novae
}

\author{
Izumi Hachisu*i \\ The University of Tokyo \\ E-mail: hachisudea.c.u-tokyo.ac.jp
}

Mariko Kato

Keio University

\begin{abstract}
We review our current understanding of multiwavelength light curves of classical and recurrent novae and show how to determine the WD masses and other binary properties. We pick up PW Vul, U Sco, V745 Sco, RS Oph, and V407 Cyg as representatives of different types of light curves, examine their light curves in detail, discuss physical properties, and clarify the reason of these differences. In the rising phase, the hydrogen-rich envelope expands beyond the size of a close binary in which the companion is embedded deep inside of the photosphere. After the optical maximum, the pseud-photosphere begins to shrink and an optically thin region develops outside of the photosphere. The free-free emission dominates the flux at relatively longer wavelengths (optical and NIR), of which light curves decay along the universal decline law of $F_{V} \propto t^{-1.75}$ (or $t^{-1.55}$ ). The physical mechanism of super-Eddington phase is presented. In the presence of shock interaction between ejecta and circumstellar matter, it slows down the decay of optical flux as $F_{v} \propto t^{-1.0}$ as seen in the early phase of V407 Cyg. In final stages of outbursts, the wind mass-loss rate sharply drops so the slope of free-free emission decays like $F_{v} \propto t^{-3.5}$. Supersoft X-ray phase begins and continues until hydrogen nuclear burning ends, and after that the nova enters a cooling phase. Hard X-rays may originate from internal shocks between ejecta (or a bow shock between ejecta and the companion). The behaviors of optical-dominant, UV-dominant, and supersoft X-ray source phase have different dependences on the WD mass and envelope chemical composition, so multiwavelength observations are useful to determine the parameters such as the WD mass. Finally, we should note that the very early phase of nova outbursts remains unexplored. An X-ray flash phase is theoretically identified, but not yet successfully detected. Detections of early X-ray flashes give direct information on thermonuclear runaway.
\end{abstract}

The Golden Age of Cataclysmic Variables and Related Objects IV

11-16 September, 2017

Palermo, Italy

\footnotetext{
*Speaker.

${ }^{\dagger}$ Dept. of Earth Science and Astronomy, College of Arts and Sciences, The University of Tokyo, Komaba, Meguroku, Tokyo 153-8902, Japan
} 


\section{Introduction}

A classical nova is a thermonuclear runaway event on a mass-accreting white dwarf (WD) in a binary. Hydrogen ignites to trigger an outburst after a critical amount of hydrogen-rich matter is accreted on the WD. The photospheric radius of the hydrogen-rich envelope expands to red-giant (RG) size and the binary becomes bright in the optical range [102, 88, 75]. The hydrogen-rich envelope then emits strong winds [54, 25]. After maximum expansion of the pseudo-photosphere, it begins to shrink, and the nova optical emission declines. Subsequently, UV emission dominates the spectrum and finally, supersoft X-ray emission increases. The nova outburst ends when the hydrogen shell burning is extinguished.

One of the recent topics on multifrequency observation is the detection of $\mathrm{GeV}$ gamma-rays from several classical novae [2]. Strong shocks of ejecta are responsible for the gamma-rays by accelerating high-energy particles. It could open a new eye on the early phase nova evolution although the origin of gamma-rays is still under debate (see, e.g., [2]). The total flux of gammarays is very small compared with (smaller than a hundredth of) the optical flux, so we may neglect the gamma-ray flux in the light curve analysis [34]. Hard X-rays may originate from internal shocks between ejecta [18, 71] (or a bow shock between ejecta and the companion [28]). The fluxes of hard X-rays are also very small compared with the optical, UV, and supersoft X-ray fluxes. In this sense, we also regard that hard X-ray emission does not affect the behavior in other wavelengths.

The main electro-magnetic wavelength region of a nova moves from optical to supersoft X-ray. We are able to estimate physical properties of a nova, such as the WD mass, by following multiwavelength behavior of a nova outburst. This is mainly because the timescale of a nova depends strongly on the WD mass [25]. In the present paper, we review observational and theoretical developments of multiwavelength behavior and obtain the physical properties of classical novae.

\section{Physics of novae as mass-accreting WDs}

\subsection{Stability of hydrogen shell-burning}

Figure 1 shows the response of WDs against various mass accretion rates on the WD mass $\left(M_{\mathrm{WD}}\right)$ vs. mass accretion rate $\left(\dot{M}_{\mathrm{acc}}\right)$ diagram (the so-called Nomoto diagram), where the accreting matter is the solar abundance, i.e., $X=0.70, Y=0.28$, and $Z=0.02$ by weight. The dashed line labeled $\dot{M}_{\text {stable }}$ is the stability line of hydrogen shell burning [57, 79, 107]. Below this line, i.e., $\dot{M}_{\text {acc }}<\dot{M}_{\text {stable }}$, hydrogen nuclear burning is unstable and no steady burning can occur. The WD suffers intermittent shell flashes, i.e., periodic nova outbursts. In the upper region of this stability line, nuclear burning is stable.

In the region between the two lines, $\dot{M}_{\text {stable }} \leq \dot{M}_{\text {acc }} \leq \dot{M}_{\text {cr }}$, we have steady hydrogen shell burning with no optically thick winds. All the accreted matter is burned into helium at the same rate as the accretion. Thus, all the matter is accumulated on the WD. The photospheric temperature of the WD envelope is sufficiently high to emit supersoft X-rays. Such objects are observed as persistent supersoft X-ray sources (SSSs) [105, 63].

In the region above the line of $\dot{M}_{\text {cr }}\left(\dot{M}_{\text {acc }}>\dot{M}_{\text {cr }}\right)$, the WD accretes matter at a higher rate than the consumption rate of hydrogen burning. The envelope expands and emits optically thick winds. Thus, the WD accretes matter via the accretion disk and a part of the accreted matter 

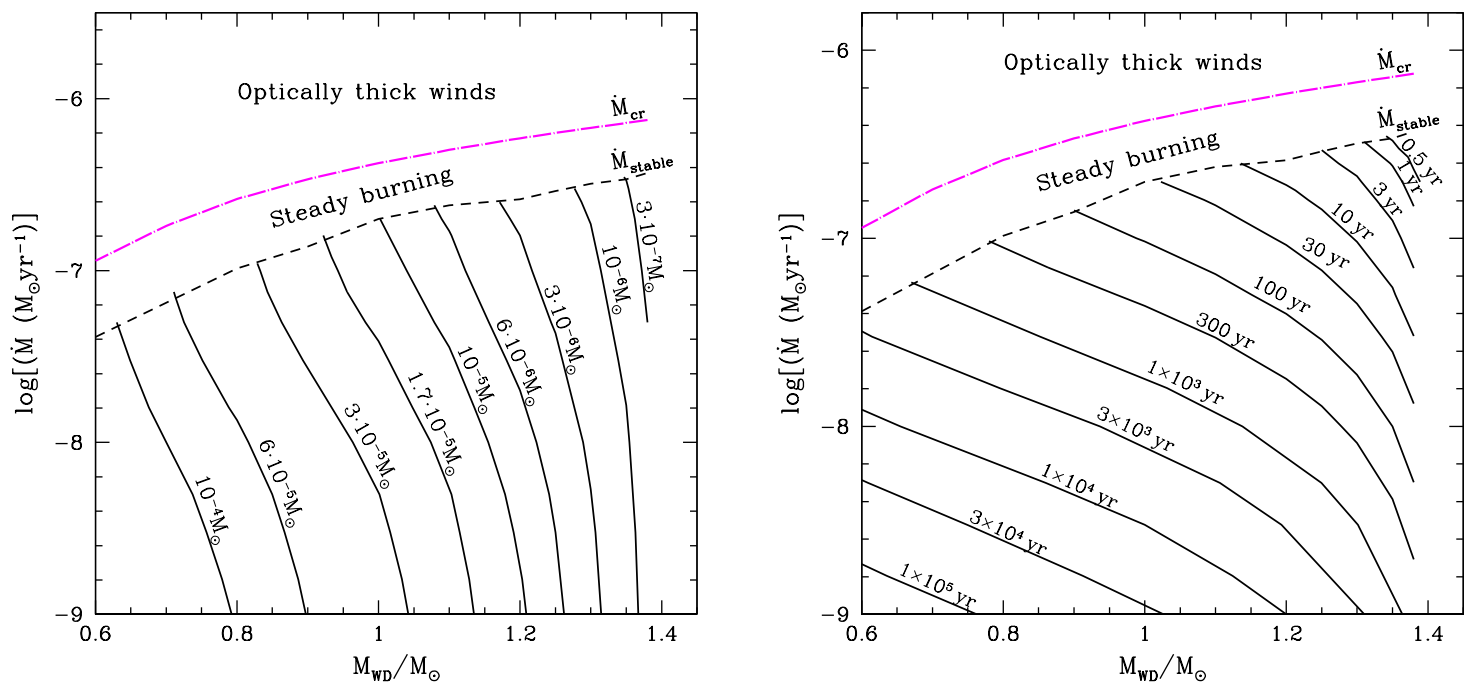

Figure 1: Left: Ignition mass $M_{\mathrm{ig}}$ on the $M_{\mathrm{WD}}-\dot{M}$ plane. The chemical composition of accreting matter is solar $(X=0.70, Y=0.28$, and $Z=0.02$ by weight). Hydrogen burning is stable in the region above the

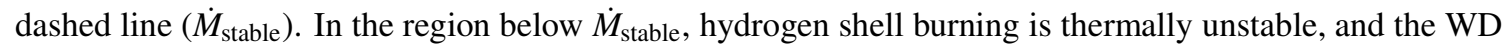
experiences shell flashes. Black solid lines indicate equi-ignition masses, the values of which are shown beside each line. The WD emits optically thick winds in the region above the dash-dotted line $\left(\dot{M}_{\mathrm{cr}}\right)$. Right: Recurrence period $t_{\text {rec }}$ of novae on the $M_{\mathrm{WD}}-\dot{M}$ plane. We plot the loci of the equi-recurrence periods of novae (black solid lines). Other symbols/lines are the same as in the left figure. The data are taken from Figures 3 and 6 of Kato et al. (2014) [57].

(excess matter) is blown in the other directions at the rate of $\dot{M}_{\text {wind }} \approx \dot{M}_{\text {acc }}-\dot{M}_{\text {cr }}$. Hachisu \& Kato $([22,23])$ claimed that such accretion winds are present in the SSSs V Sge and RX J0513.9-6951. Their light curve models reasonably reproduce the cyclic behavior of the optical/X-ray light curves with the cyclic on/off behavior of the accretion winds (intermittent SSS phase). Such binaries are considered as objects corresponding to the accretion wind phase. To summarize, the three $\dot{M}_{\text {acc }}$ regions in Figure 1, that is, the upper accretion wind (optically thick wind) region, mid steady burning region, and lower nova region have corresponding objects mentioned above, that is, the intermittent SSSs, persistent SSSs, and classical novae.

\subsection{Physics of wind mass loss}

Binary WDs suffer mass loss both in the accretion wind evolution and nova outburst. We present the mechanisms of wind mass-loss and summarize their nature. Figure 2 shows a structure of a hydrogen-rich envelope on a 1.0 $M_{\odot}$ WD when the envelope expands to red-giant size after ignition. Here, we assume steady state of the hydrogen-rich envelope, in which $\dot{M}_{r}=4 \pi r^{2} \rho v=$ constant along the radius $r$ [54]. The horizontal axis is the radius from the WD center. Hydrogen shellburning is taking place at the bottom of the hydrogen-rich envelope and produces nuclear energy as shown by the local luminosity of $L_{r}$. The luminosity exceeds the local Eddington luminosity of $L_{\text {Edd }}=4 \pi c G M_{r} / \kappa$ at $\log r(\mathrm{~cm}) \sim 10.8$. The matter is accelerated in this super-Eddington region and the velocity $v$ eventually exceeds the escape velocity $v_{\text {esc }}$. The open blue square indicates the 


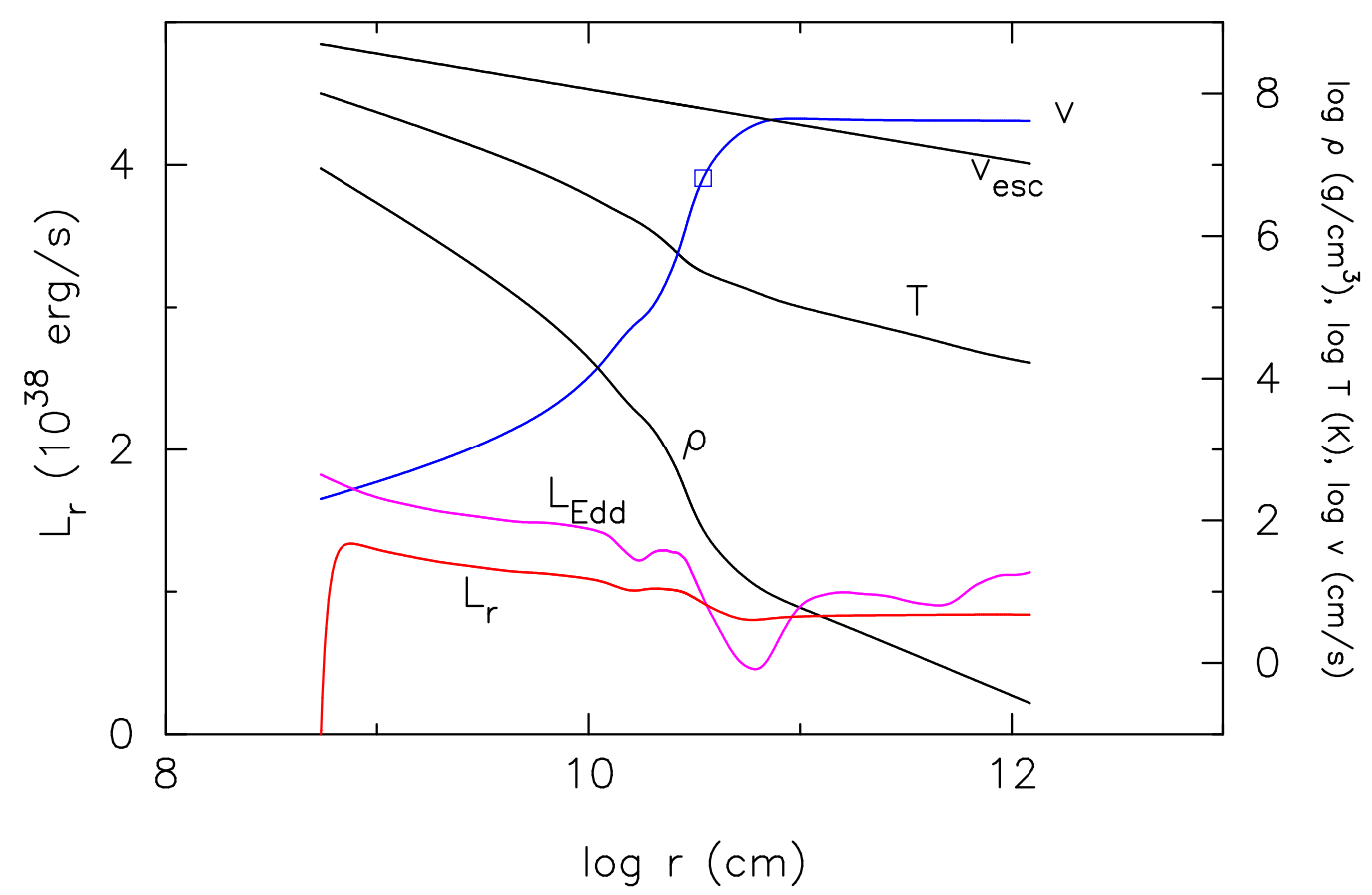

Figure 2: Structure of a hydrogen-rich envelope on a $1.0 M_{\odot}$ WD when the envelope expands to red-giant size after ignition. The WD emits strong winds. The abscissa is the radius from the WD center. Hydrogen shell-burning is taking place at the bottom of the hydrogen-rich envelope and produces nuclear energy as shown by the local luminosity of $L_{r}$ (solid red line). The luminosity exceeds the local Eddington luminosity of $L_{\text {Edd }}$ (solid magenta line) at $\log r(\mathrm{~cm}) \sim 10.8$. Matter is accelerated in this super-Eddington region and the velocity $v$ (solid blue line) eventually exceeds the escape velocity $v_{\text {esc }}$. The open blue square indicates the sonic point. The data are taken from [54].

sonic point. The evolution of a nova outburst can be computed using these optically thick wind model [54].

\subsection{Nova optical and near-infrared light curves}

Optical and near-infrared (NIR) light curves of novae can be calculated from the photospheric luminosity in the very early phase of the outburst. Figure 3 shows a schematic illustration of nova envelope just after the outburst. The density of the envelope is relatively dense so that the photosphere exists at/near the surface of nova extended envelope as shown in Figure 3(a). As the envelope expands largely and emits strong winds, the photosphere detaches the surface of the hydrogen-rich envelope and, as a result, an optically thin region develops as shown in Figure 3(b). The optical flux is dominated by free-free emission from the optically thin plasma, so the optical light curve can be calculated from the wind mass-loss rate [25], that is,

$$
F_{V} \propto \int N_{\mathrm{e}} N_{\mathrm{i}} d V \propto \int_{R_{\mathrm{ph}}}^{\infty} \frac{\dot{M}_{\mathrm{wind}}^{2}}{v_{\mathrm{wind}}^{2} r^{4}} r^{2} d r \propto \frac{\dot{M}_{\mathrm{wind}}^{2}}{v_{\mathrm{ph}}^{2} R_{\mathrm{ph}}}
$$

during the optically thick wind phase, where $F_{v}$ is the flux at the frequency $v, N_{\mathrm{e}}$ and $N_{\mathrm{i}}$ the number densities of electron and ion, respectively, $V$ the volume of the ejecta, $\dot{M}_{\text {wind }}$ the wind massloss rate 
(a) pre-maximum

\section{fire-ball phase}

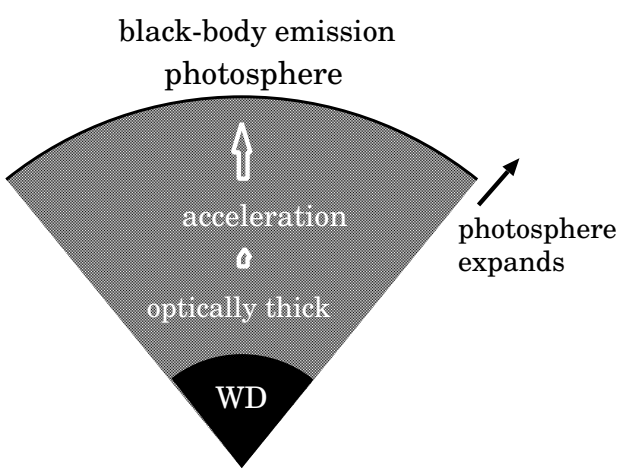

(b) post-maximum

\section{free-free emission phase}

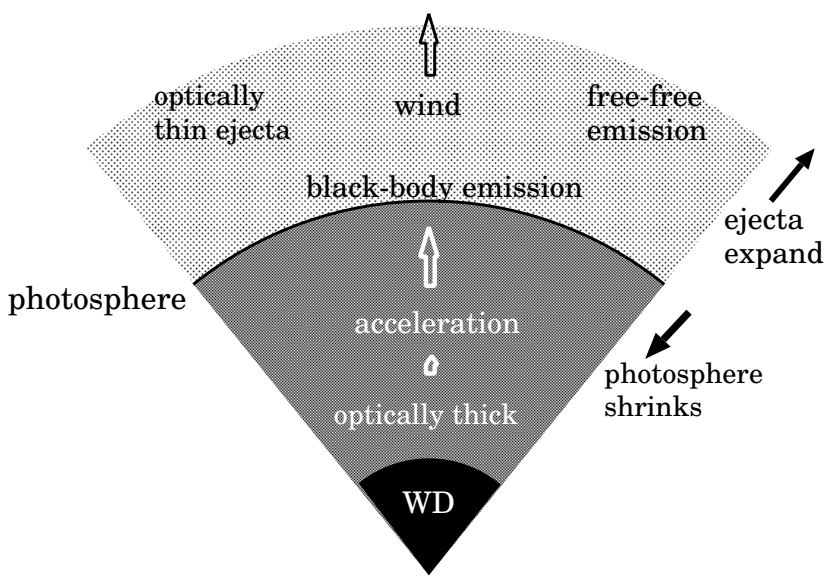

Figure 3: Schematic configuration of hydrogen-rich envelope on the WD after ignition. (a) The density of the hydrogen-rich envelope is relatively high so that the photosphere moves almost together with the surface of the envelope. We observe the photospheric emission (approximated by black-body emission). (b) As the envelope expands largely, the photosphere detaches the surface of the hydrogen-rich envelope and, as a result, an optically thin region develops. Free-free emission from the optically thin plasma dominates the spectrum. Reproduced from [31].

[25]. Here, we use the relation of $\rho_{\text {wind }}=\dot{M}_{\text {wind }} / 4 \pi r^{2} v_{\text {wind }}$, and $\rho_{\text {wind }}$ and $v_{\text {wind }}$ are the density and velocity of the wind, respectively. After the wind stops, we obtain

$$
F_{V} \propto \int N_{\mathrm{e}} N_{\mathrm{i}} d V \propto \rho^{2} V \propto \frac{M_{\mathrm{ej}}^{2}}{V^{2}} V\left(\propto R^{-3} \propto t^{-3}\right),
$$

[108, 25], where $\rho$ is the density, $M_{\mathrm{ej}}$ the ejecta mass (in parenthesis, if $M_{\mathrm{ej}}$ is constant in time), $R$ the radius of the ejecta $\left(V \propto R^{3}\right)$, and $t$ the time after the outburst. We calculate $\dot{M}_{\text {wind }}, R_{\mathrm{ph}}, M_{\mathrm{ej}}$, and so on, from the optically thick wind solutions as shown in Figure 2.

Figure 4 shows a schematic illustration of nova evolution after a nova explosion sets in. (a) The photosphere expands greatly and reaches maximum. Free-free emission dominates the spectrum. The companion star is engulfed deep inside the photosphere. (b) After the maximum expansion, the photospheric radius shrinks with time. (c) A large part of the envelope matter is blown in the wind and the photosphere moves further inside. (d) The companion eventually emerges from the WD photosphere and an accretion disk may appear or reestablished again. (e) The photosphere further shrinks to a size of $\lesssim 0.1 R_{\odot}$. (f) The optically thick wind stops. (g) Hydrogen nuclear burning stops and the nova enters a cooling phase. The optical flux dominates the spectra from stage (a) to (b). The ultraviolet (UV) flux dominates from stage (b) to (f). The supersoft X-ray flux replaces the UV flux from stage (f) to (g). (see e.g., Hachisu \& Kato 2006 [25]). 
(a)

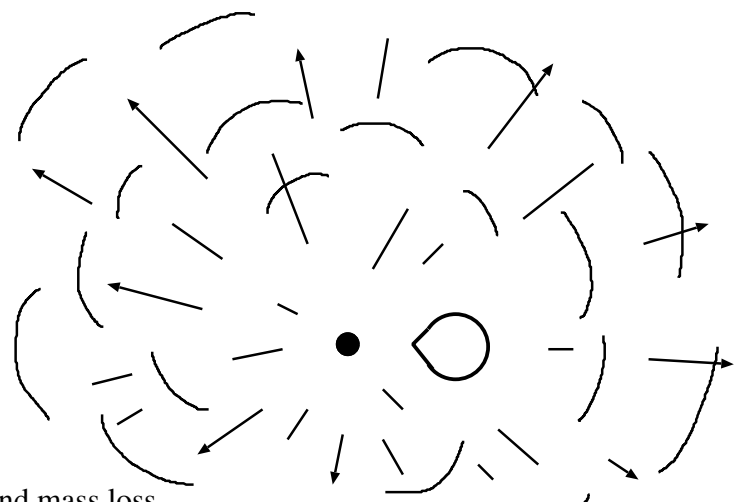

(b)

wind mass loss

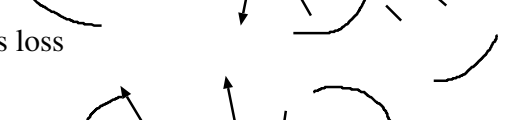

(c)

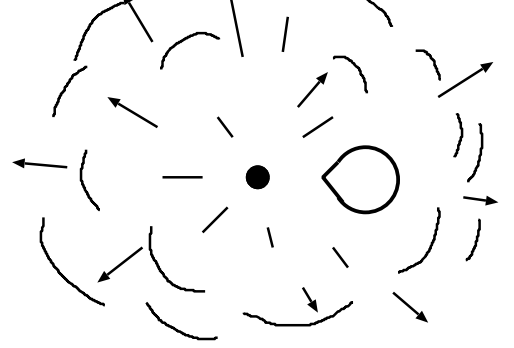

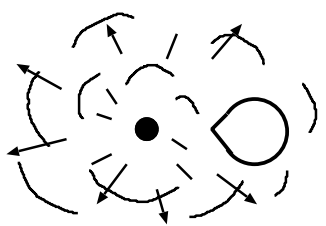

(d)

$$
\begin{aligned}
& \text { companion } \\
& \text { re-appears }
\end{aligned}
$$

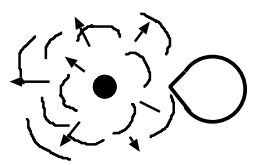

(e)

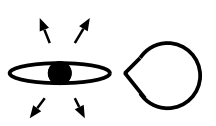

wind stops



(g)
stops

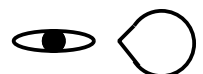

Supersoft

X-ray

Figure 4: Schematic illustration of a nova evolution: (a) after a nova explosion sets in, the photosphere expands greatly and the companion star is engulfed deep inside the photosphere; (b) after the maximum expansion, the photospheric radius shrinks with time and free-free emission dominates the flux at relatively longer wavelengths; (c) a large part of the envelope matter is blown in the wind and the photosphere moves further inside; (d) the companion eventually emerges from the WD photosphere and an accretion disk may appear or reestablished again; (e) the photosphere further shrinks to a size of $\lesssim 0.1 R_{\odot}$; (f) the optically thick wind stops; (g) hydrogen nuclear burning stops and the nova enters a cooling phase. Hard X-rays may originate from internal shocks between ejecta (or a bow shock between ejecta and the companion) from stage (a) to (f) as indicated by a dashed line. The optical flux dominates the spectra from stage (a) to (b). The ultraviolet (UV) flux dominates from stage (b) to (f). Then the supersoft X-ray flux replaces the UV flux from stage (f) to $(\mathrm{g})$. The figure is reproduced from Figure 1 of [25]. 


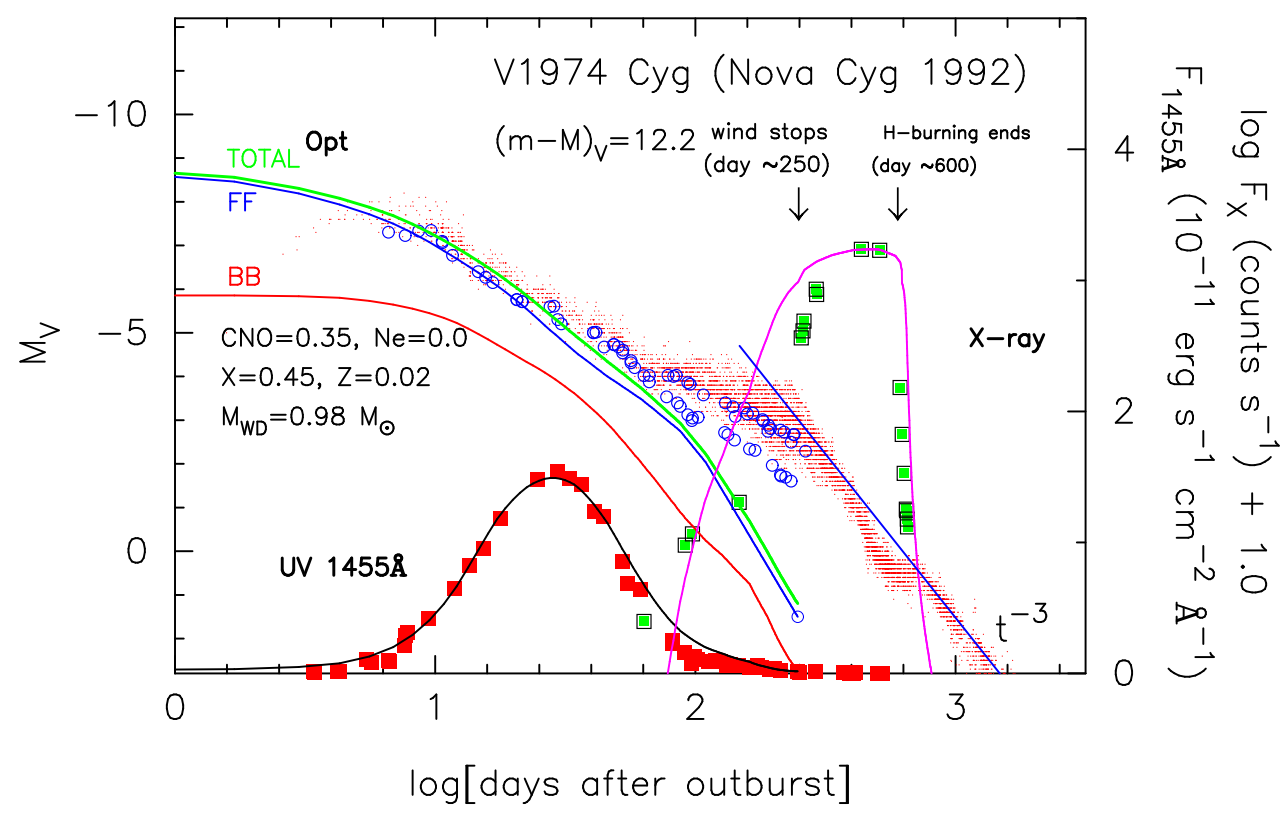

Figure 5: Multiwavelength light curves of the classical nova V1974 Cyg. The $V$ (blue open circles), visual (red dots), UV $1455 \AA$ (filled red squares), and supersoft X-ray (filled green squares with black outlines) fluxes. We assume that the distance modulus in the $V$ band is $(m-M)_{\mathrm{V}}=12.2(d=1.8 \mathrm{kpc}$ and $E(B-V)=$ 0.30 , see [32]). Then the optical $V$ magnitude exceeds the Eddington limit $\left(M_{V \text {,Edd }} \approx-5.8\right.$ for $1.0 M_{\odot}$, see Equation (3) of [24]) by about $1.7 \mathrm{mag}$. The duration of super-Eddington phase is about 17 days. The solid lines represent the theoretical multiwavelength light curves of $M_{\mathrm{WD}}=0.98 M_{\odot}$ with the chemical composition of $X=0.45, Y=0.18, X_{\mathrm{CNO}}=0.35$, and $Z=0.02$ by weight. The total optical flux (green line labeled "TOTAL") is the summation of the contribution of the free-free emission (blue line labeled "FF") from the outflowing winds and the blackbody emission (red line labeled "BB") at the photosphere. The $t^{-3}$ line indicates the trend of free-free flux for a freely expanding nebula with no mass supply. The optically thick wind stops at day $\sim 250$ and hydrogen burning extinguishes at day $\sim 600$. This figure is reproduced from data in Hachisu \& Kato (2016a) [32].

Figure 5 shows an example of light curves of classical novae taken from Hachisu \& Kato (2016a) [32]. The durations of the optically bright and UV bright phases are governed by the massloss rate, which depends strongly on the WD mass and weakly on the chemical composition of the envelope. Here, we plot the UV $1455 \AA$ band flux, which is a narrow band with $20 \AA$ width at the central wavelength of $1455 \AA$ invented by Cassatella et al. (2002) [8]. The supersoft X-ray phase also depends, although differently, on this WD mass and chemical composition. The optical spectra of novae are basically free-free emission, so the optical light curve is calculated with the wind massloss rate. The ultraviolet (narrow $1455 \AA$ band) and supersoft X-ray fluxes are calculated assuming blackbody emission from the photosphere. The optical $V$, UV $1455 \AA$, and supersoft X-ray fluxes are well reproduced with the $0.98 M_{\odot}$ WD model with the chemical composition of $X=0.45$, $Y=0.18, X_{\mathrm{CNO}}=0.35$, and $Z=0.02$ by weight.

\subsection{Physics of super-Eddington luminosity}

Many novae have a super-Eddington phase in their early stages. Its peak luminosity often ex- 


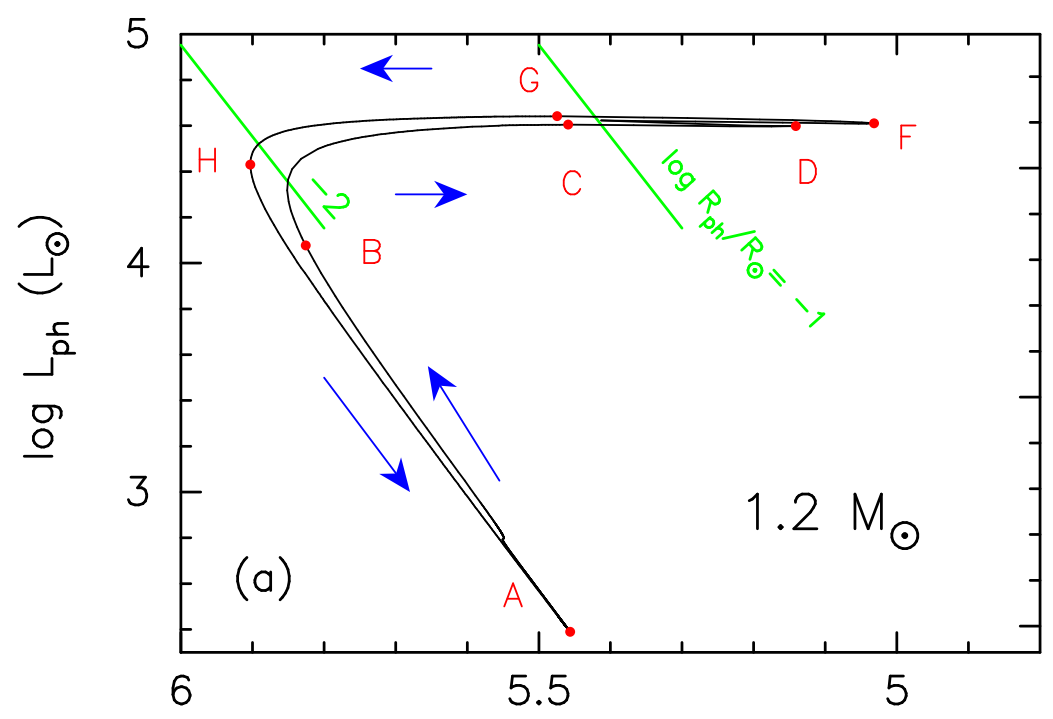

38

$\bar{\circ}$

$\frac{2}{2}$

37 ลิ

os

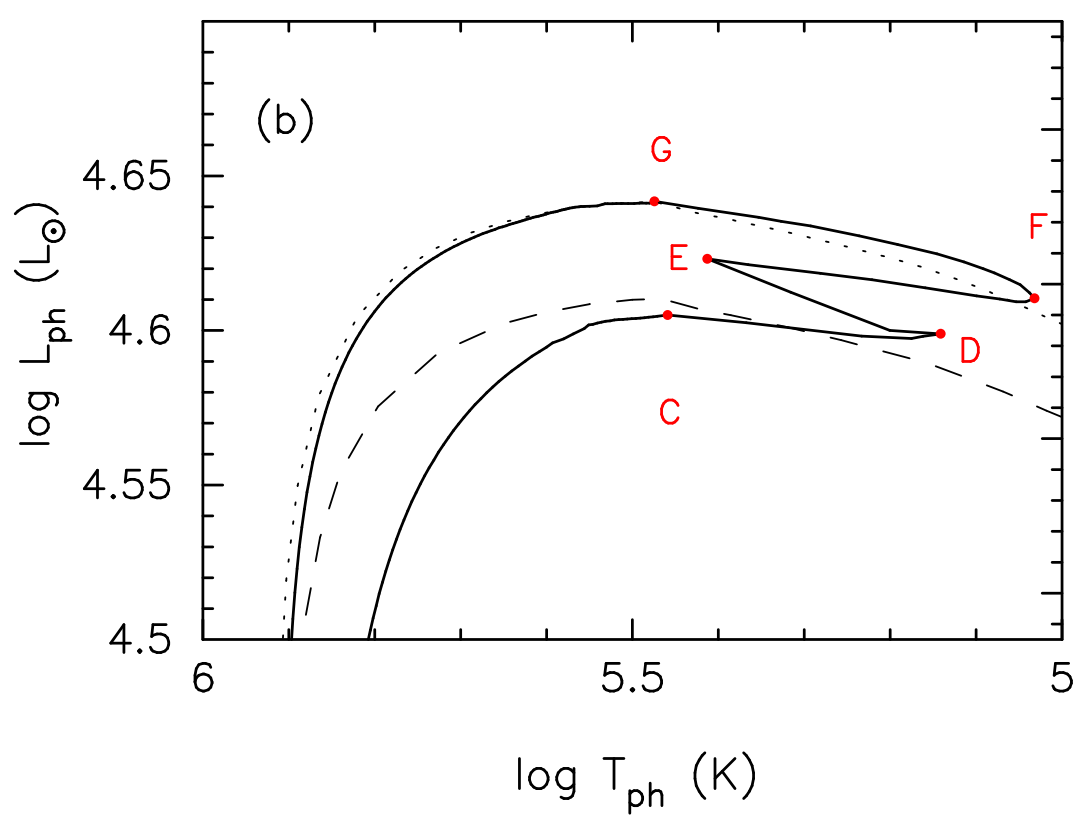

38.25

$\overline{0}$

38.2 욱

$\frac{\widehat{D}}{6}$

38.15 $\stackrel{1}{\sim}$

38.1

Figure 6: Evolution track of a hydrogen shell flash (black line) in the HR diagram for a $1.2 M_{\odot}$ WD. The accreted matter is solar $(X=0.70, Y=0.28$, and $Z=0.02$ by weight). The mass accretion rate is $2.0 \times$ $10^{-7} M_{\odot} \mathrm{yr}^{-1}$ and the recurrence period is $9.9 \mathrm{yr}$. (a) The entire scale is shown while (b) an enlargement of the track. The blue arrows indicate the direction of evolution. The thin solid green lines indicate the lines of $\log R_{\mathrm{ph}} / R_{\odot}=-2$ and -1 . The optically thick wind blows in the region right to point $\mathrm{C}$ (or point $\mathrm{G}$ ). The small zig-zag path between $\log T(\mathrm{~K}) \sim 5.5$ and 5.0 is due to the change in chemical composition, i.e., the surface layer with the original composition of accreted matter is blown away in the wind. The data are taken from [58]. 

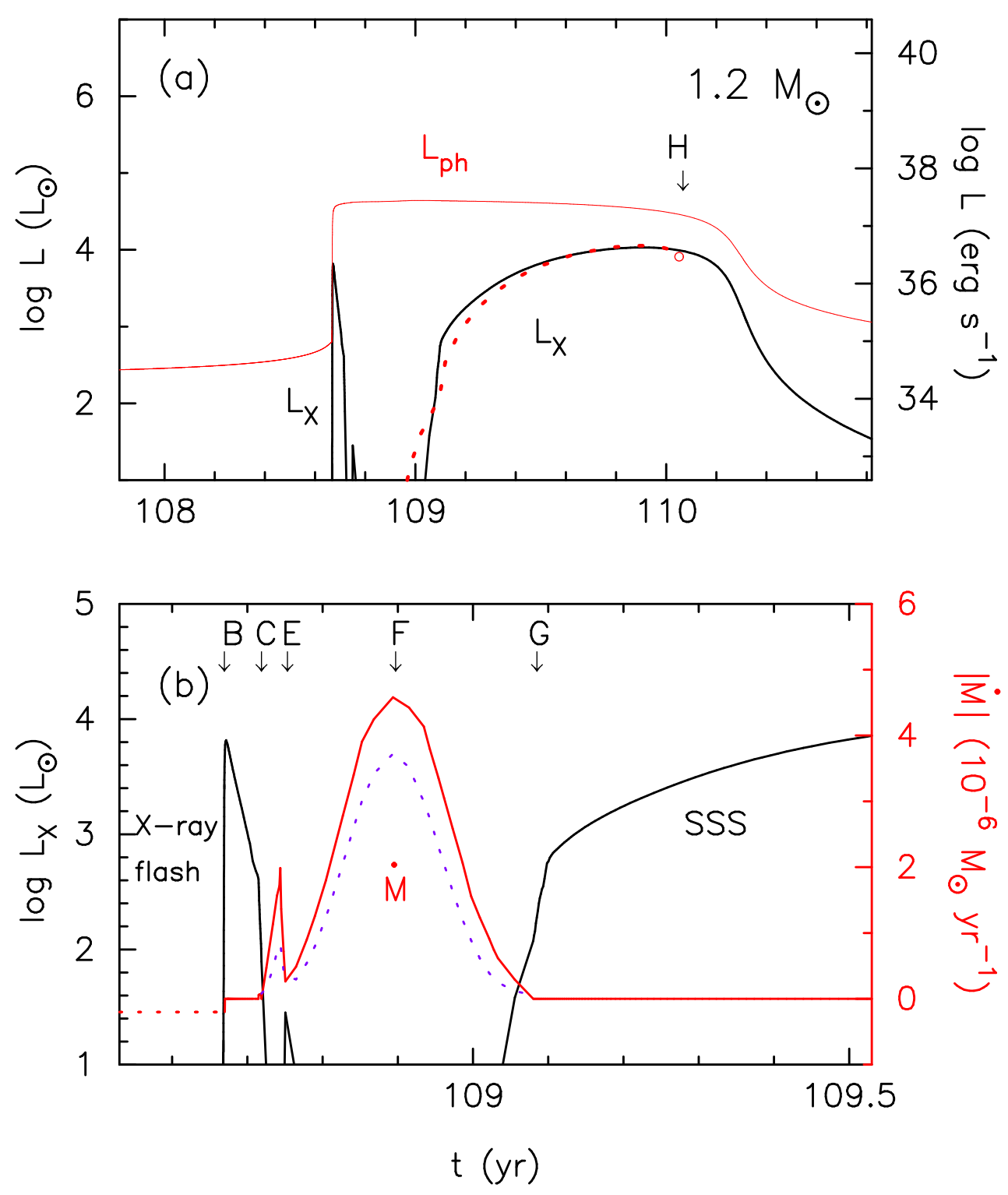

Figure 7: Evolution calculation of a nova on a $1.2 M_{\odot}$ WD. The same model as in Figure 6. The data are taken from [58]. The solid black lines show the supersoft X-ray flux $\left(0.3-1.0 \mathrm{keV}\right.$, denoted by $\left.L_{X}\right)$. (a) The entire phase of a hydrogen shell-flash. We also plot the photospheric (bolometric) luminosity $L_{\mathrm{ph}}$ by the solid red line. (b) An enlargement of the hydrogen shell-flash including the wind mass-loss rate $(\dot{M}$, solid red line). 
ceeds the Eddington limit at the photosphere by a factor of a few to several. The duration of superEddington phase is sometimes longer than several days. The physics of the super-Eddington stage is theoretically often misunderstood, so we explain here the mechanisms of the super-Eddington luminosity.

The classical nova V1974 Cyg has a super-Eddington phase, of which the peak luminosity exceeds the Eddington luminosity by $1.7 \mathrm{mag}$ in $V$ band and the duration lasts about 17 days (see Figure 5). Here, we assume that the distance and reddening toward V1974 Cyg are $d=$ $1.8 \mathrm{kpc}$ and $E(B-V)=0.30$ [32]. Then the distance modulus in $V$ band is $\mu_{V} \equiv(m-M)_{V}=$ 12.2. The Eddington limit for a $1.0 M_{\odot}$ star is $M_{V, \text { Edd }}=-5.8$ from Equation (3) of [24]. The optical $V$ light curve is well reproduced by the total flux (green line, labeled "TOTAL"), which is the sum of free-free emission (blue line, labeled "FF" outside the photosphere) and photospheric blackbody emission (red line, labeled "BB" at the photosphere). Note that the optical emission from the blackbody photosphere is slightly sub-Eddington, while the optical free-free emission reaches super-Eddington luminosities.

The emissivity of the free-free emission is proportional to the square of the wind mass-loss rate, i.e., $F_{v} \propto \dot{M}_{\text {wind }}^{2}$ from Eq.(2.1). The free-free flux can exceed the Eddington value when the wind mass-loss rate is sufficiently high in the early phase. This is because the flux of free-free emission originates from optically thin region and has nothing to do with the Eddington limit. The mass-loss rate decreases as the photospheric temperature increases with time, so the free-free emission light curve decays with time. The optically thick winds can reproduce a number of nova light curves including early super-Eddington phases.

\subsection{HR diagram of novae}

Figure 6 shows the HR diagram of a $1.2 M_{\odot}$ WD model. Here, we assume the solar composition of accreted matter and the mass accretion rate of $2.0 \times 10^{-7} M_{\odot} \mathrm{yr}^{-1}$. The resultant recurrence period is $9.9 \mathrm{yr}$. In the HR diagram the bolometric luminosity $L_{\mathrm{ph}}$ is almost constant in the bright phase and does not exceed the Eddington luminosity of electron scattering, that is, $L_{\mathrm{Edd}, \mathrm{el}}=4 \pi c G M_{\mathrm{WD}} / \kappa_{\mathrm{el}}$ and $\kappa_{\mathrm{el}}=0.2(1+X) \mathrm{cm}^{2} \mathrm{~g}^{-1}$ is the opacity for electron scattering. Figure 7 shows the evolution of the same nova as in Figure 6. This property that $L_{\mathrm{ph}}$ does not exceeds $L_{\text {Edd,el }}$ can be seen in any other papers that show the HR diagrams for one nova outburst cycle (e.g., $[49,62,12,58,59])$, being independent of the old/new opacities. This is because the large nuclear luminosity produced in the nuclear burning region is quickly absorbed in a layer around the nuclear burning zone and, as a result, the outward photon flux is sub-Eddington [58].

\subsection{X-ray flash and supersoft X-ray source phase}

Figure 7 also depicts the evolution of X-ray light curves, which is the same model as in Figure 6. There are two supersoft X-ray bright phases. We can observe the first supersoft X-ray bright phase in the very early phase of a nova outburst even if it is very short. We can also observe the second supersoft X-ray bright phase in the later phase of the nova outburst, the duration of which is rather long compared with the first one. We call the first one the X-ray flash and the second one the supersoft X-ray source (SSS) phase. No X-ray flash has been observed, although a few tasks were attempted $[60,67]$. 


\section{Multifrequency observations of novae}

In this section, we present several novae as examples of typical multifrequency study of novae.

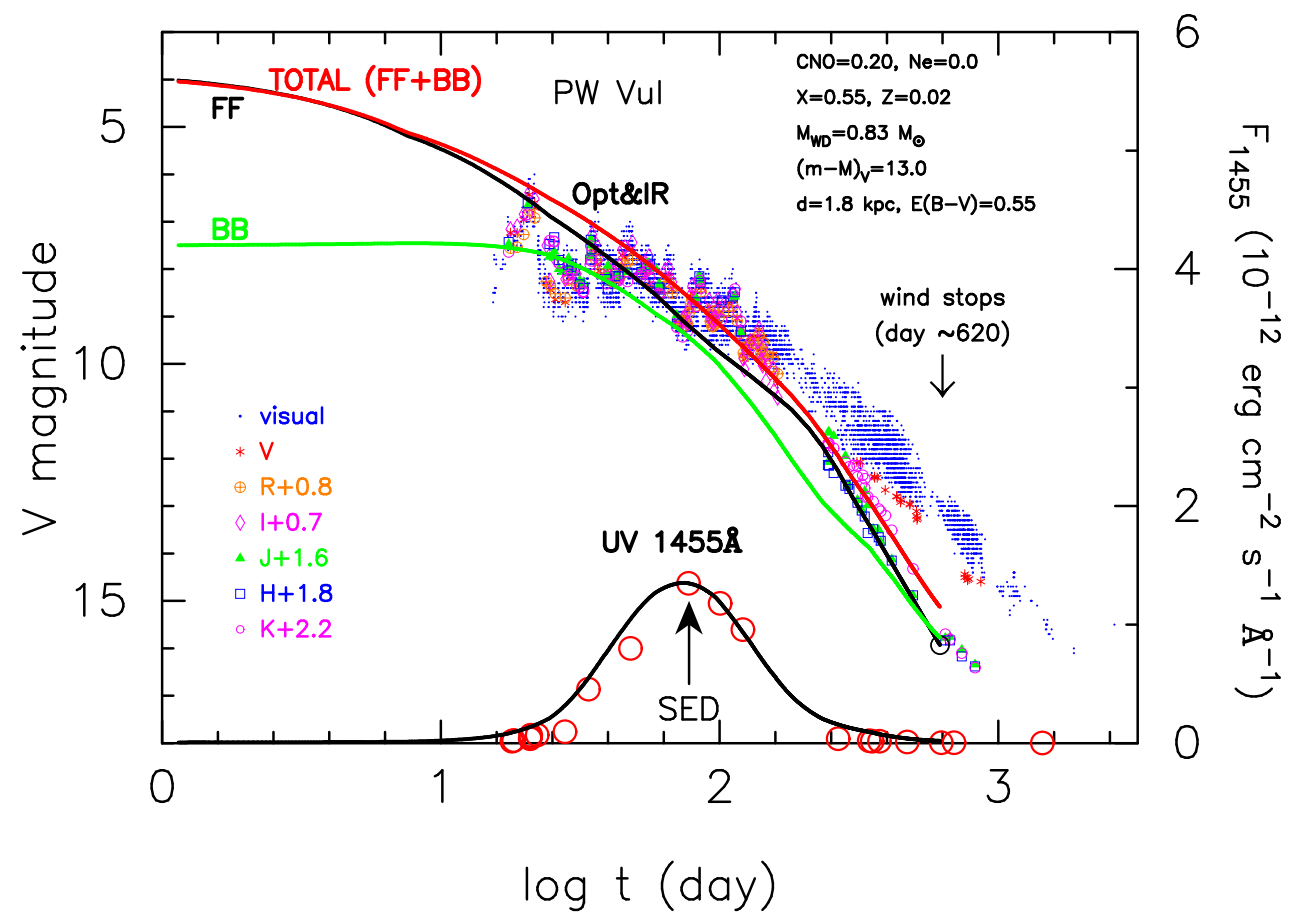

Figure 8: Model light curve fitting to the observed fluxes of PW Vul. The black solid line labeled "FF" shows the $V$ magnitude calculated from free-free emission. The green solid line labeled "BB" denotes the $V$ magnitude calculated from the photospheric blackbody emission. The red solid line labeled "TOTAL" represents the total $V$ magnitude (BB plus FF). The distance modulus in $V$ band is $(m-M)_{V}=13.0$. The total $V$ light curve is in reasonable agreement with the $V$ observation (red asterisks) until the nebular phase started. The visual magnitudes (blue dots), which are taken from the AAVSO archive, are also added. The model $V$ light curve reasonably fits with the early optical $V$ light curve until the nebular phase started, but deviates from the observation in the nebular phase. This deviation is due to strong emission lines such as [O III], because the model flux does not include emission lines. The spectrum in Figure 9 was secured at the day denoted by an arrow labeled "SED."

\subsection{PW Vul}

PW Vul (Nova Vulpeculae 1984 \#1) was discovered by Wakuda on UT 1984 July 27.7 about a week before its optical maximum of $m_{V, \max }=6.3$ on UT 1984 August 4.1. The optical, NIR, and UV $1455 \AA$ light curves are plotted in Figure 8 on a logarithmic timescale. The X-ray flux increased during the first year [80] and faded before the ROSAT observation $(1990-1999)$. No X-ray data are available in the supersoft $\mathrm{X}$-ray source phase.

Andreae et al. (1991) [3] obtained $E(B-V)=0.58 \pm 0.06$ from HeII $\lambda 1640 / \lambda 4686$ ratio and $E(B-V)=0.55 \pm 0.1$ from the interstellar absorption feature at $2200 \AA$ for the reddening toward PW Vul. Saizar et al. (1991) [91] reported $E(B-V)=0.60 \pm 0.06$ from HeII $\lambda 1640 / \lambda 4686$ ratio. Duerbeck et al. (1984) [15] estimated the extinction to be $E(B-V)=$ 




Figure 9: Spectrum of PW Vul 64 days after the outburst (Day $0=$ UT 1984 Sep $30=$ JD 2445 973.5) dereddened with $E(B-V)=0.55$. Thin red solid line: IUE spectra, SWP24088 and LWP04458, are taken from the INES data archive server. Open red circles: $U B V R I$ data from [90] and JHKLM data from [19]. Global features of spectrum can be fitted with a combination (thick black solid line) of the blackbody with a temperature of $T_{\mathrm{BB}}=27,000 \mathrm{~K}$ (thick magenta line) and the optically-thick, free-free emission with the same electron temperature of $T_{\mathrm{e}}=27,000 \mathrm{~K}$ (thick blue line). We also add a straight solid red line of $F_{\lambda} \propto \lambda^{-8 / 3} \approx \lambda^{-2.67}$.

Table 1: Selected model chemical compositions of novae

\begin{tabular}{llllllll}
\hline Nova case & $X$ & $Y$ & $X_{\mathrm{CNO}}$ & $X_{\mathrm{Ne}}$ & $Z$ & Mixing & Object \\
\hline CO nova 1 & 0.35 & 0.13 & 0.50 & 0.0 & 0.02 & $100 \%$ & DQ Her \\
CO nova 2 & 0.35 & 0.33 & 0.30 & 0.0 & 0.02 & $100 \%$ & GQ Mus \\
CO nova 3 & 0.45 & 0.18 & 0.35 & 0.0 & 0.02 & $55 \%$ & V1668 Cyg \\
CO nova 4 & 0.55 & 0.23 & 0.20 & 0.0 & 0.02 & $25 \%$ & PW Vul \\
Ne nova 1 & 0.35 & 0.33 & 0.20 & 0.10 & 0.02 & $100 \%$ & V351 Pup \\
Ne nova 2 & 0.55 & 0.30 & 0.10 & 0.03 & 0.02 & $25 \%$ & V1500 Cyg \\
Ne nova 3 & 0.65 & 0.27 & 0.03 & 0.03 & 0.02 & $8 \%$ & QU Vul \\
Solar & 0.70 & 0.28 & 0.0 & 0.0 & 0.02 & $0 \%$ & \\
\hline
\end{tabular}


$0.45 \pm 0.1$ from galactic extinction in the direction toward the nova, whose galactic coordinates are $(l, b)=\left(61.0983^{\circ},+5.1967^{\circ}\right)$. For the galactic extinction, we examined the galactic dust absorption map in the NASA/IPAC Infrared Science Archive ${ }^{1}$, which is calculated on the basis of data from Schlafly et al. (2011) [95]. It gives $E(B-V)=0.43 \pm 0.02$ in the direction of PW Vul. The arithmetic mean of these four values is $E(B-V)=0.55 \pm 0.05$. Recently, Hachisu \& Kato (2014) [30] proposed a new method for determining reddening of classical novae. They identified a general course of $U B V$ color-color evolution and determined reddenings of novae by matching the track of a target nova with their general course. They obtained $E(B-V)=0.55 \pm 0.05$ for PW Vul, which agrees well with the above mean value. Therefore, we adopt $E(B-V)=0.55 \pm 0.05$.

As for the distance to PW Vul, a reliable estimate, $d=1.8 \pm 0.05 \mathrm{kpc}$, was obtained by Downes \& Duerbeck (2000) [14] through the nebular expansion parallax method. Adopting this value, together with $E(B-V)=0.55 \pm 0.05$, the distance modulus of PW Vul is

$$
\begin{aligned}
(m-M)_{V} & =5 \log \left(\frac{d}{10 \mathrm{pc}}\right)+3.1 E(B-V) \\
& =5 \log (180 \pm 5)+3.1(0.55 \pm 0.05)=13.0 \pm 0.2
\end{aligned}
$$

Therefore, we adopt the distance modulus in $V$ band of $(m-M)_{V}=13.0 \pm 0.2$ for PW Vul.

One of the most intriguing properties of classical novae is metal-enrichment of ejecta (see, e.g., Gehrz et al. (1998) [20]), which is ascribed to mixing with WD core material during outburst (see, e.g., [89]). PW Vul is not an exception of this general trend, as summarized in Table 1 of Hachisu \& Kato (2014) [30]. There is a noticeable scatter in the abundance estimates, from $X=0.47$ to $X=0.69$. Their arithmetic mean is $X=0.57, Y=0.22$, and $X_{\mathrm{CNO}}=0.19$ (for fixed $Z=0.02$ ). Here, $X, Y, Z$, and $X_{\mathrm{CNO}}$ are hydrogen, helium, heavy elements with the solar abundance, and carbon-nitrogen-oxygen fractions in weight, respectively.

Hachisu \& Kato $(2006,2010,2015)[25,28,31]$ took a simple parameterization for the degree of mixing between core material and accreted matter as $\eta_{\text {mix }}=(0.7 / X)-1$. Here we assume the solar composition for the accreted matter. Table 1 lists seven representative cases of Hachisu \& Kato's degree of mixing, that is, $100 \%$ (denoted by "CO nova 1", "CO nova 2", and "Ne nova 1"), $55 \%$ ("CO nova 3"), 25\% ("CO nova 4" and "Ne nova 2"), and 8\% ("Ne nova 3"). We adopt CO nova 4 for PW Vul, because this composition is closest to the averaged values of PW Vul among the above seven in Table 1.

Hachisu \& Kato $(2014,2015)[30,31]$ analyzed the spectra of PW Vul assuming that the continuum flux $F_{V}$ is simply the sum of a blackbody spectrum of the photospheric temperature $T_{\mathrm{ph}}$ and an optically thick free-free emission with the electron temperature of $T_{\mathrm{e}}$, i.e.,

$$
F_{v}=f_{1} B_{v}\left(T_{\mathrm{ph}}\right)+f_{2} S_{v}\left(T_{\mathrm{e}}\right)
$$

where $v$ is the frequency, $B_{v}\left(T_{\mathrm{ph}}\right)$ is the Planckian for the photospheric temperature $T_{\mathrm{ph}}$, and $S_{v}\left(T_{\mathrm{e}}\right)$ is the optically thick free-free spectrum for the electron temperature $T_{\mathrm{e}}, f_{1}$ and $f_{2}$ are numeric constants (e.g., [77]). Following Wright \& Barlow (1975) [109], the optically thick free-free spectrum can be expressed as

$$
S_{v}\left(T_{\mathrm{e}}\right)=B_{v}\left(T_{\mathrm{e}}\right) K_{v}^{2 / 3}\left(T_{\mathrm{e}}\right)
$$

\footnotetext{
${ }^{1}$ http://irsa.ipac.caltech.edu/applications/DUST/
} 
where the linear free-free absorption coefficient $K_{v}\left(T_{\mathrm{e}}\right)$ is given by

$$
K_{v}\left(T_{\mathrm{e}}\right)=3.7 \times 10^{8}\left[1-\exp \left(-\frac{h v}{k T_{\mathrm{e}}}\right)\right] Z^{2} g_{v}\left(T_{\mathrm{e}}\right) T_{\mathrm{e}}^{-1 / 2} v^{-3}
$$

in cgs units; $g_{v}\left(T_{\mathrm{e}}\right)$ is the Gaunt factor. In general, the Gaunt factor depends weakly on the frequency and temperature, but we assume it to be unity following Hachisu \& Kato (2014) [30]. So there are four fitting parameters, i.e., $f_{1}, f_{2}, T_{\mathrm{ph}}=T_{\mathrm{BB}}$, and $T_{\mathrm{e}}$. When $h v \ll k T_{e}$, Wright $\&$ Barlow (1975) [109] derived

$$
S_{v}=23.2\left(\frac{\dot{M}_{\text {wind }}}{\mu v_{\infty}}\right)^{4 / 3} \frac{v^{2 / 3}}{D^{2}} \gamma^{2 / 3} g^{2 / 3} Z^{4 / 3} \mathrm{Jy},
$$

where $1 \mathrm{Jy}=10^{-26} \mathrm{~W} \mathrm{~m}^{-2} \mathrm{~Hz}^{-1}$; the ion number density is assumed equal to the total gas number density $n$ and the electron number density is equal to $\gamma$ times the ion number density; $\dot{M}_{\text {wind }}$ is the wind mass-loss rate in units of $M_{\odot} \mathrm{yr}^{-1} ; D$ is the distance in units of $\mathrm{kpc} ; v_{\infty}$ is the terminal wind velocity in units of $\mathrm{km} \mathrm{s}^{-1} ; \mu$ is the mean molecular weight; $Z$ is the charged-degree of ion (only in this formula); $v$ is the frequency in units of $\mathrm{Hz}$; and $g$ is the Gaunt factor. Equation (3.5) can be further simplified as

$$
S_{v} \propto v^{2 / 3}, \text { or } S_{\lambda} \propto \lambda^{-8 / 3},
$$

where $\lambda$ is the wavelength.

Figure 9 shows the contributions of $B_{\lambda}$ and $S_{\lambda}$ in a spectrum of PW Vul. When the wind mass loss rate is small, contribution of free-free emission is relatively small in Equations (3.2) and (3.5). Hachisu \& Kato (2014) [30] decomposed the spectrum of PW Vul about 64 days after the outburst using Equation (3.2) and concluded that the free-free flux $S_{\lambda}$ is comparable to the pseudo-photospheric flux $B_{\lambda}$ in $V$ band. We can see that the blackbody emission gives a good approximation to the UV $1455 \AA$ region and the free-free emission is a good fit to the infrared (IR) region. In the region between them, we have a comparable contribution from the blackbody and free-free components. The decomposition in Figure 9 indicates that the photospheric emission may substantially contribute in the optical light curve.

Figure 8 also shows the theoretical light curves, from the sum of free-free plus blackbody emission for the optical bands, and from only the blackbody emission for the UV $1455 \AA$ band. The $0.83 M_{\odot}$ WD model (black thin solid lines) shows reasonable agreement with the optical, NIR, and UV observations, in particular with the UV $1455 \AA$ observations [31]. An arrow labeled SED indicates the date (Day 64) at which the spectrum in Figure 9 was secured. The $0.83 M_{\odot}$ WD model has $T_{\mathrm{ph}}=28,000 \mathrm{~K}$ on this day, which is close to and consistent with the blackbody temperature of $T_{\mathrm{BB}}=27,000 \mathrm{~K}$ determined by the decomposition in Figure 9 .

From the UV $1455 \AA$ light curve fitting of the $0.83 M_{\odot}$ WD in Figure 8, Hachisu \& Kato (2015) [31] obtained the following distance-reddening relation, i.e.,

$$
\begin{aligned}
& 2.5 \log F_{\lambda 1455}^{\mathrm{mod}}-2.5 \log F_{\lambda 1455}^{\mathrm{obs}} \\
= & 2.5 \log \left(3.38 \times 10^{-12}\right)-2.5 \log \left((1.35 \pm 0.27) \times 10^{-12}\right) \\
= & 5 \log \left(\frac{d}{10 \mathrm{kpc}}\right)+8.3 \times E(B-V),
\end{aligned}
$$

where $F_{\lambda 1455}^{\text {mod }}=3.38 \times 10^{-12} \mathrm{erg} \mathrm{cm}^{-2} \mathrm{~s}^{-1} \AA^{-1}$ is the calculated UV $1455 \AA$ band flux at maximum of the $0.83 M_{\odot}$ WD model for an assumed distance of $10 \mathrm{kpc}$ and $F_{\lambda 1455}^{\mathrm{obs}}=(1.35 \pm 0.27) \times$ 
$10^{-12} \mathrm{erg} \mathrm{cm}^{-2} \mathrm{~s}^{-1} \AA^{-1}$ is the maximum observed flux [8]. Here they assumed an absorption of $A_{\lambda}=8.3 \times E(B-V)$ at $\lambda=1455 \AA$ taken from [96]. Two distance-reddening relations of Equations (3.1) and (3.7) cross each other at the point of $(E(B-V), d)=(0.57 \pm 0.05 \mathrm{mag}, 1.75 \pm 0.3 \mathrm{kpc})$, being consistent with the observations, i.e., $E(B-V)=0.55 \pm 0.05$ and $d=1.8 \pm 0.05 \mathrm{kpc}$, summarized above. These good agreements suggest that the WD mass of PW Vul is as massive as $\sim 0.83 M_{\odot}$ if the chemical composition is close to $X=0.55, Y=0.23, Z=0.02$, and $X_{\mathrm{CNO}}=0.20$.

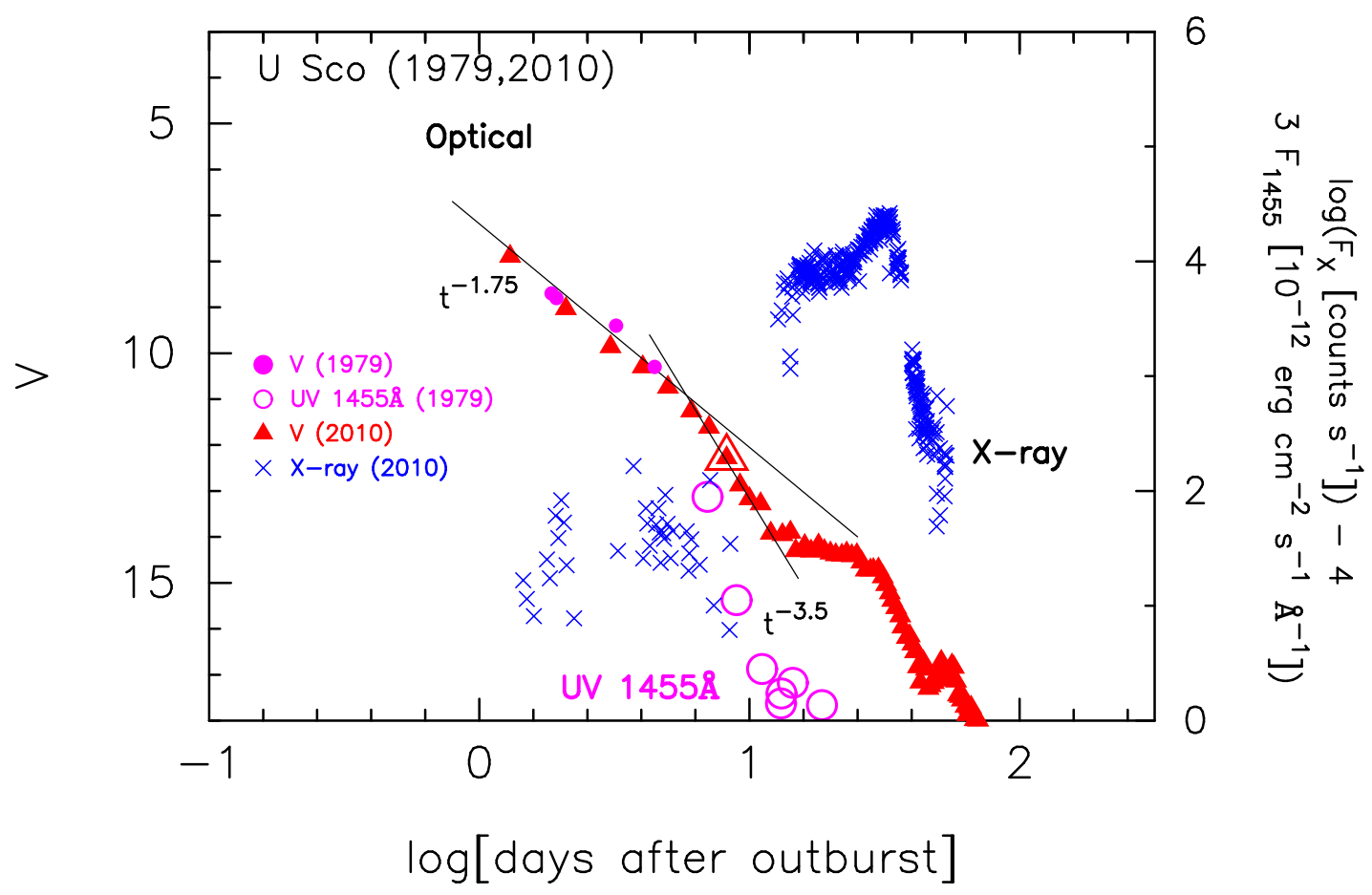

Figure 10: Multifrequency light curves of U Sco for the 1979 and 2010 outbursts. The filled red triangles denote the $V$ magnitudes of the U Sco (2010) outburst, and the filled and open magenta circles represent the $V$ and UV $1455 \AA$ fluxes of the U Sco (1979) outburst, respectively. The blue crosses are the X-ray $(0.3-10$ $\mathrm{keV}$ ) count rates of the U Sco (2010) outburst. The large red triangle indicates the start of the nebular phase approximately eight days after the outburst.

\subsection{U Sco}

U Sco is a recurrent nova with ten recorded outbursts in 1863, 1906, 1917, 1936, 1945, 1969, 1979, 1987, 1999, and 2010, nearly every ten years [93]. Figure 10 shows the $V$, UV $1455 \AA$, and X-ray light curves of the U Sco $(1979,2010)$ outbursts on a logarithmic timescale. The $V$ data of the U Sco (2010) outburst are taken from [85]. The X-ray data of the U Sco (2010) outburst are from the Swift web page [17]. The $V$ data of the U Sco (1979) outburst are from IAU Circular No.3373(2) and the UV $1455 \AA$ fluxes of the U Sco (1979) outburst are calculated using the data taken from the INES archive data sever ${ }^{2}$. The maximum brightness and decline rates of the 2010 outburst were estimated to be $m_{V, \max }=7.6, t_{2}=1.7$ days, and $t_{3}=3.6$ days by [94].

$\mathrm{U}$ Sco is one of the fastest novae. The WD mass should be very massive and close to the Chandrasekhar mass. Its mass was estimated by Thoroughgood et al. (2001) [103] to be $M_{\mathrm{WD}}=$

\footnotetext{
${ }^{2}$ http://sdc.laeff.inta.es/
} 
$1.55 \pm 0.24 M_{\odot}$ from the primary orbital velocity of $K_{\mathrm{W}}=93 \pm 10 \mathrm{~km} \mathrm{~s}^{-1}$ and the secondary orbital velocity of $K_{\mathrm{R}}=170 \pm 10 \mathrm{~km} \mathrm{~s}^{-1}$. Hachisu et al. (2000) [36] obtained the WD mass of $M_{\mathrm{WD}}=1.37 \pm 0.01 M_{\odot}$ for the U Sco (1999) outburst based on their model light curve fitting. Using the $1.37 M_{\odot}$ WD model, Hachisu \& Kato (2012) [29] reasonably reproduced the $V$ and supersoft X-ray light curves for the U Sco (2010) outburst. Thus, we regard the WD mass of U Sco to be $1.37 M_{\odot}$. Hachisu et al. (2000) [36] argued that U Sco is a promising progenitor of SNe Ia.

The slope of nova decay changes from the universal decline of $F_{V} \propto t^{-1.75}$ [25] to a more rapid decay of $F_{V} \propto t^{-3.5}$ by approximately eight days after the outburst, at the large open red triangle, as shown in Figure 10. We call this change of slope the break. Such a change of decay trend can be seen in the model light curves (see, e.g., Figure 49 of [35]) when the wind mass-loss rate sharply decreases and the photosphere quickly shrinks. In the case of V1668 Cyg, this change is coincident with the start of the nebular phase, as shown in Figure 49 of [35]. Therefore, we consider this change as the transition to optically thin from optically thick of the ejecta.

Approximately 14 days after the outburst, the $V$ light curve of $U$ Sco becomes flat, as shown in Figure 10. This plateau is due to the contribution from the irradiated accretion disk [36]. Approximately 30 days after the outburst, hydrogen shell-burning ended, as clearly shown by the drop in supersoft X-ray flux in Figure 10, which corresponds to the end of the plateau phase in the $V$ light curve.

Hachisu \& Kato (2018b) [35] obtained $(m-M)_{V}=16.3 \pm 0.2$ and $E(B-V)=0.26 \pm 0.05$ $(d=12.6 \pm 2.0 \mathrm{kpc})$ from various light curve fittings.

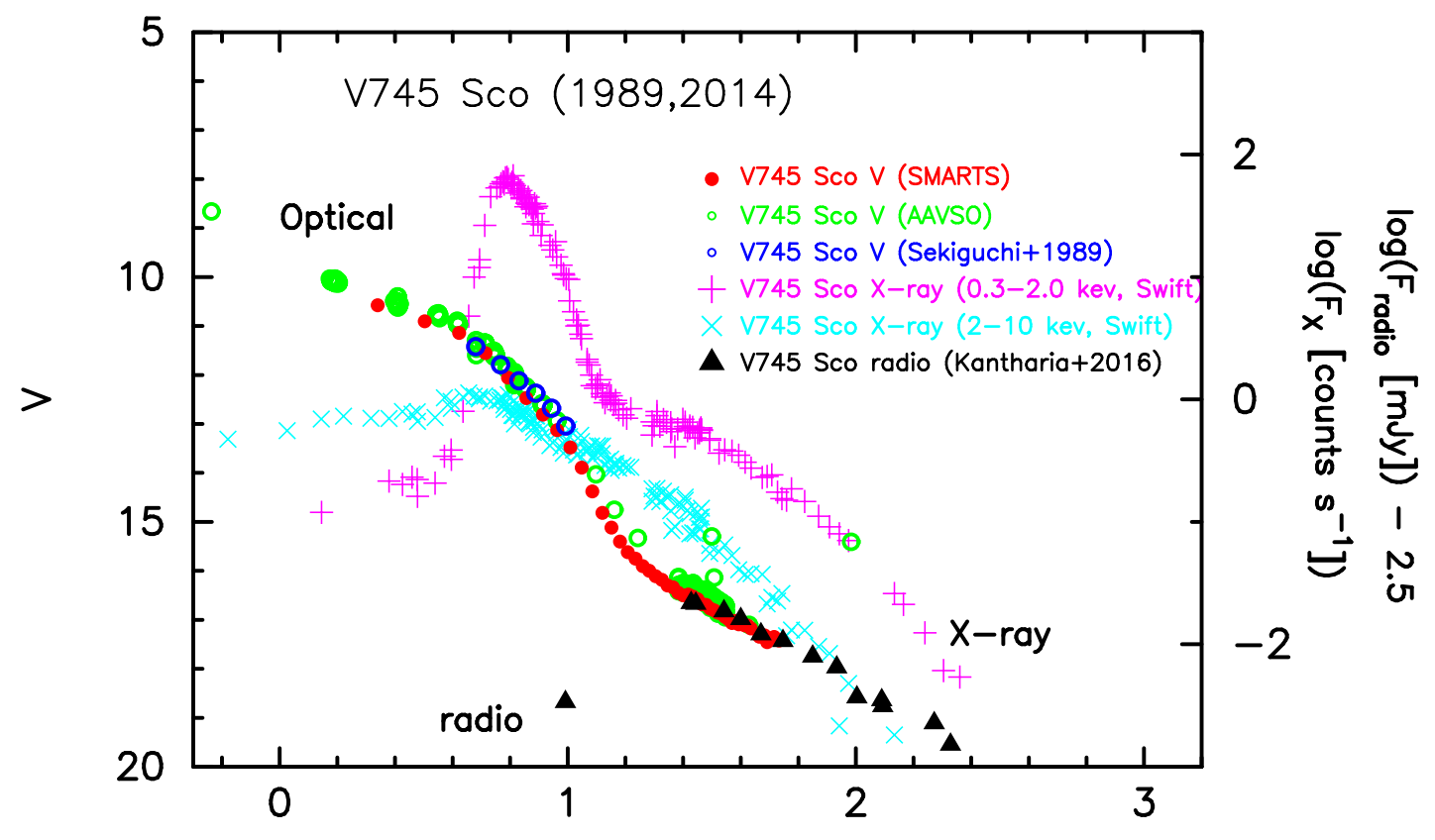

Figure 11: Multifrequency light curves of V745 Sco for the 1989 and 2014 outbursts. The $V$ light curve of the V745 Sco 1989 (open blue circles, taken from [97]) and 2014 (filled red circles from SMARTS and open green circles from AAVSO) outbursts are plotted on a logarithmic timescale. We also include X-ray $(0.3-2.0 \mathrm{keV}$ with magenta pluses and $2.0-10 \mathrm{keV}$ with cyan crosses) data taken from [84] and radio (filled black triangles, GMRT at $610 \mathrm{MHz}$ ) data taken from [53]. 


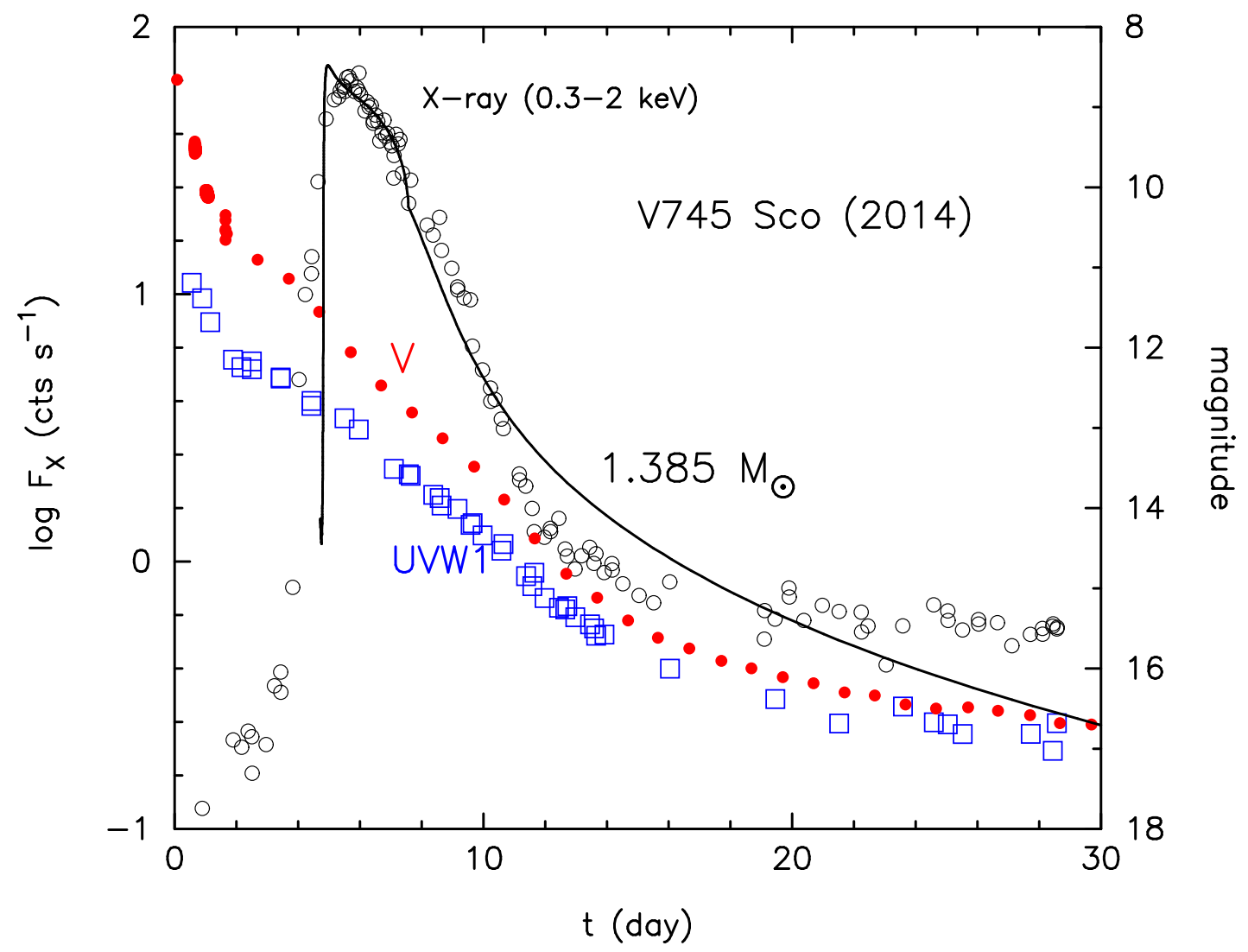

Figure 12: Model light curve fitting of the supersoft $\mathrm{X}$-ray flux $(0.3-2.0 \mathrm{keV}$ denoted by open black circles) for the V745 Sco (2014) outburst. The data are the same as those in Figure 7 of [35]. The start of day (horizontal axis) is the discovery date (JD 2456695.194). We also include the $V$ (filled red circles) and UVW1 (open blue squares) magnitudes. All the observational data are taken from [84]. A $1.385 M_{\odot}$ WD model (solid black line) reasonably reproduces the observed supersoft X-ray flux.

\subsection{V745 Sco}

V745 Sco is a recurrent nova with three recorded outbursts in 1937, 1989, and 2014 [84]. Figure 11 shows the $V$ light curve on a logarithmic timescale. The 2014 outburst shows $m_{V, \max }=$ 8.66 (AAVSO), $t_{2}=2$ days, and $t_{3}=4$ days (e.g., [82]).

The distance to V745 Sco was estimated by Schaefer (2009) [92] to be $d=7.8 \mathrm{kpc}$ from the orbital period of $P_{\text {orb }}=510 \pm 20$ days and the corresponding Roche lobe size. However, this period was not confirmed by Mróz et al. (2014) [69], and thus, we do not use the method based on the Roche lobe size.

Mróz et al. (2014) [69] detected semi-regular pulsations of the RG companion (with periods of 136.5 days and 77.4 days). Hachisu \& Kato (2016b) [33] estimated the distance from the empirical period-luminosity relation for pulsating red-giants, i.e.,

$$
M_{K}=-3.51 \times(\log P(\text { day })-2.38)-7.25 \text {, }
$$

with an error of $\sim 0.2 \mathrm{mag}$ [106]. Hachisu \& Kato (2016b) [33] obtained the absolute $K$ magnitude of $M_{K}=-6.39 \pm 0.2$ for the fundamental 136.5-day pulsation from the data of [69]. Adopting an 
average $K$ mag of $m_{K}=8.33$ mag [47], Hachisu \& Kato (2016b) [33] obtained $d=7.8 \pm 0.8 \mathrm{kpc}$ for $E(B-V)=0.70$ from

$$
(m-M)_{K}=0.353 \times E(B-V)+5 \log (d / 1 \mathrm{kpc})+10,
$$

where they adopted the reddening law of $A_{K}=0.353 \times E(B-V)$ [7]. Their new distance is accidentally identical to Schaefer's value of $7.8 \mathrm{kpc}$. The vertical distance from the galactic plane is approximately $z=-540 \mathrm{pc}$, being significantly above the scale height of galactic matter distribution $(z= \pm 125$ pc, see, e.g., [64]). We may conclude that V745 Sco belongs to the galactic bulge because the galactic coordinates are $(l, b)=\left(357.3583^{\circ},-3.9989^{\circ}\right)$.

The SSS phase of V745 Sco started just four days after the discovery (Figure 12). If the optical brightness reaches its maximum at the discovery date, this turn-on time of SSS is the earliest record ( $t_{\mathrm{SSS}-\mathrm{on}}=4$ days) among novae. The second shortest record $\left(t_{\mathrm{SSS}-\mathrm{on}}=6\right.$ days $)$ is the 1 -yr recurrence period nova, M31N 2008-12a [45]. Kato et al. (2017) [58] modeled time-evolutions of such very-short-timescale novae and obtained a reasonable light curve fit with a $1.38 M_{\odot}$ WD for the $1 \mathrm{yr}$ recurrence period nova M31N 2008-12a. The earlier appearance of SSS for V745 Sco suggests a more massive WD than that of M31N 2008-12a. Hachisu \& Kato (2018b) [35] modeled time-evolutions of novae similar to the calculation of [58] and obtained a reasonable fit (solid black line) with the supersoft X-ray light curve (open black circles), as shown in Figure 12. We plot the supersoft X-ray flux $(0.3-2.0 \mathrm{keV}$ count rates of the Swift XRT) as well as the $V$ magnitude and Swift UVW1 magnitude light curves. All the observational data are taken from [84]. Hachisu \& Kato (2018b) [35] obtained the WD mass to be $1.385 M_{\odot}$, more massive than $1.38 M_{\odot}$ for M31N 2008-12a. It is unlikely that these WDs were born as massive as they are $\left(1.38 M_{\odot}\right.$ or $1.385 M_{\odot}$, see, e.g., [13]). We suppose that these WDs have grown in mass. Nomoto (1982) [78] showed that, if a WD has a carbon-oxygen core and its mass reaches $\sim 1.38 M_{\odot}$ or more, the WD explodes as a SN Ia. Therefore, V745 Sco is one of the most promising candidates of SN Ia progenitors.

\subsection{RS Oph}

RS Oph is a recurrent nova with six recorded outbursts in 1898, 1933, 1958, 1967, 1985, and 2006 (e.g., [93]). The orbital period of 453.6 days was obtained by Brandi et al. (2009) [6]. Figure 13 shows the $V$ magnitudes ([10], [99], AAVSO, SMART), radio (1.49 GHz) fluxes [46], UV $1455 \AA$ [8], and X-ray [38] light curves of RS Oph on a logarithmic timescale. The WD mass of RS Oph was estimated to be $M_{\mathrm{WD}}=1.35 \pm 0.01 M_{\odot}$ by Hachisu et al. $(2006,2007)[37,38]$ from the model $V$ and supersoft X-ray light curve fittings. Thus, we regard the WD mass of RS Oph to be $1.35 M_{\odot}$. Hachisu \& Kato (2001) [21] argued that RS Oph is a progenitor of SNe Ia because the WD mass is close to the SN Ia explosion mass of $M_{\text {Ia }}=1.38 M_{\odot}$ (e.g., [78]) and now increases (see also [40, 41, 42, 65]).

The $V$ and radio light curves clearly show the trend of $F_{V} \propto t^{-1.55}$ as shown in Figure 13. The slope of $F_{v} \propto t^{-1.55}$ is close to the slope of universal decline law proposed by Hachisu \& Kato (2006) [25]. However, the SMEI magnitude light curve has a different trend of $L_{\mathrm{SMEI}} \propto t^{-1.0}$, where $L_{\text {SMEI }}$ is the luminosity of the SMEI band. This is because the SMEI magnitude is a wide-band (peak quantum efficiency at $700 \mathrm{~nm}$ with a full width at half maximum (FWHM) of $300 \mathrm{~nm}$ ) and 




Figure 13: Multifrequency light curves of RS Oph for the 1958, 1985, and 2006 outbursts. The $V$ data (filled red circles) are taken from [99], AAVSO, and SMARTS for the 2006 outburst and also $V$ from [10] but for the 1958 outburst. The SMEI band data (filled cyan circles) are from [48] for the 2006 outburst. We also plot the supersoft X-ray $(0.3-0.55 \mathrm{keV}$, magenta plus, taken from [38]) for the 2006 outburst, UV $1455 \AA$ band (filled blue triangles, taken from [8]) and radio $(1.49 \mathrm{GHz})$ band fluxes (filled green stars, taken from [46]) both for the 1985 outburst.

include the flux of very strong $\mathrm{H} \alpha$ line, which mainly comes from the shock interaction. RS Oph has a RG companion and the companion star emits cool slow winds $\left(\sim 40 \mathrm{~km} \mathrm{~s}^{-1}\right.$, see [50]), which form circum-stellar matter (CSM) around the binary before the nova outburst. The ejecta of the nova outburst have high velocity, up to $\sim 4000 \mathrm{~km} \mathrm{~s}^{-1}$, and collide with the CSM, giving rise to strong shock (e.g., [101]). The shock interaction contributes to the $\mathrm{H} \alpha$ line and slows the decay of SMEI magnitude. Such an interaction between ejecta and CSM was frequently observed in supernovae Type IIn, and the relation $L_{V} \propto t^{-1.0}$ in the $V$-band luminosity was calculated by Moriya et al. (2013) [68] for SN 2005ip. We discuss this point in more detail in the next subsection on V407 Cyg.

For the reddening and distance modulus toward RS Oph, we adopt $E(B-V)=0.65 \pm 0.05$ and $(m-M)_{V}=12.8 \pm 0.2$ after Hachisu \& Kato (2016b) [33]. The distance is calculated to be $d=1.4 \pm 0.2 \mathrm{kpc}$. This reddening is roughly consistent with those obtained by Snijders (1987) [100], i.e., $E(B-V)=0.73 \pm 0.06$ from the HeII line ratio of $1640 \AA$ and $3203 \AA$, and $E(B-V)=$ $0.73 \pm 0.10$ from the $2715 \AA$ interstellar dust absorption feature. The NASA/IPAC galactic dust absorption map also gives $E(B-V)=0.64 \pm 0.03$ in the direction toward RS Oph, whose galactic coordinates are $(l, b)=\left(19.7995^{\circ},+10.3721^{\circ}\right)$. 
There are still debates on the distance to RS Oph (see, e.g., [93]). Hjellming et al. (1986) [46] estimated the distance to be $1.6 \mathrm{kpc}$ from Hi absorption-line measurements. Snijders (1987) [100] also obtained the distance of $1.6 \mathrm{kpc}$ assuming the UV peak flux is equal to the Eddington luminosity. Harrison et al. (1993) [43] calculated a distance of $1290 \mathrm{pc}$ from the $K$-band luminosity. Hachisu \& Kato (2006b) [37] obtained the distance to be $1.3-1.7 \mathrm{kpc}$ from the $y$ and $I_{c}$ band light curve fittings in the late phase of the 2006 outburst. O'Brien et al. (2006) [81] estimated the distance of $1.6 \mathrm{kpc}$ from VLBA mapping observation with an expansion velocity indicated from emission line width. Barry et al. (2008) [5] reviewed various estimates and summarized that, for the 2006 outburst, the canonical distance is $1.4_{-0.2}^{+0.6} \mathrm{kpc}$. Here, we adopt $E(B-V)=0.65 \pm 0.05$ and $d=1.4 \pm 0.2 \mathrm{kpc}$, which give the distance modulus in $V$ band of $(m-M)_{V}=12.8 \pm 0.2$.



Figure 14: Optical and X-ray light curves of V407 Cyg together with RS Oph. The filled red circles denote the $V$ magnitudes of V407 Cyg, and the open blue squares represent the $V$ magnitudes of RS Oph. The $V$ data of V407 Cyg are taken from [73] and [16]. We add the $I_{\mathrm{C}}$ magnitudes (open magenta diamonds taken from AAVSO and VSOLJ) of V407 Cyg and the SMEI magnitudes (filled blue circles) of RS Oph. We also add the X-ray fluxes of V407 Cyg (red pluses, taken from [76]) and RS Oph (open blue stars).

\subsection{V407 Cyg}

V407 Cyg is a well-observed symbiotic nova (e.g., [72]), in which a WD accretes mass from a cool RG companion via the Roche-lobe overflow or stellar wind. The RG companion of V407 Cyg is a Mira-like variable with the pulsation period of 762.9 days in the $K$ band [61]. SiO maser observations suggest that the Mira-like companion has already reached a very late evolution stage of an AGB star [11,9]. The 2010 outburst of V407 Cyg was discovered on UT 2010 March 10.813 at $m_{V}=7.6$. Figure 14 shows the $V$ and X-ray light curves of V407 Cyg on a logarithmic timescale. The nova reached $m_{V, \max }=7.1$ on UT 2010 March 10.8 (JD 2455266.3) and then 
declined with $t_{2}=5.9$ days and $t_{3}=24$ days in the $V$-band [73]. The $V$ light curve sharply drops around JD 2455310 about 44 days after the optical maximum.

X-rays were observed with Swift, which are interpreted in terms of strong shock heating between the ejecta and circumstellar cool wind [76]. The strong shock possibly produced $\mathrm{GeV}$ gamma-rays by accelerating high-energy particles. V407 Cyg is the first gamma-ray-detected nova with Fermi/Large Area Telescope (LAT) [1] (see Figure 14). Since V407 Cyg, GeV gammarays were observed in six novae between 2010 and 2015, i.e., V1324 Sco, V959 Mon, V339 Del, V1369 Cen, V745 Sco, and V5668 Sgr (e.g., [2, 70]).

\subsubsection{Reddening and distance}

Many authors adopted the reddening of $E(B-V)=0.57$ and the distance of $d=2.7 \mathrm{kpc}$ after Munari et al. (1990) [72], who derived $E(B-V)=0.57$ by fitting the broad-band spectrum of V407 Cyg with an M6III model spectrum and $d=2.7 \mathrm{kpc}$ from the Mira-like stars' periodluminosity relation. Shore et al. (2011) [98] derived $E(B-V)=0.45 \pm 0.05$ from the depth of the diffuse interstellar absorption bands and proposed that the Mira-like star looks like an M8III rather than M6III type. Iijima (2015) [51] argued that the diffuse interstellar bands cannot give a reliable reddening because the different bands resulted in very different values of the reddening; for example, $E(B-V)=0.15$ from the band at $\lambda=6613 \AA$ but $E(B-V)=0.76$ from the band at $\lambda=6270 \AA$ of his spectra. Iijima (2015) [51] obtained $E(B-V)=0.6$ from the color excess relation of $E(B-V)=(B-V)-(B-V)_{0}$ and the empirical relation of the intrinsic color of $(B-V)_{0}=-0.02 \pm 0.04$ at time $t_{2}$ [104]. He used the $B-V$ colors of VSOLJ/VSNET which are rather scattered and not reliable, as clearly shown in Figure 20 of [35]. Hachisu \& Kato (2018b) [35] adopted the $B-V$ colors of Munari et al. (2011) [73] and the empirical relation of [104] and obtained the reddening to be $E(B-V) \sim 1.0$ (at $t_{2}$-time). Therefore, we adopt $E(B-V)=1.0$ after Hachisu \& Kato (2018b) [35].

V407 Cyg is a symbiotic binary star system consisting of a mass-accreting hot WD and a cool Mira-like giant with a pulsation period of 762.9 days in the $K$ band [61]. The period-luminosity relation of the LMC Mira-like stars has a bend at the pulsation period of $\sim 400$ days [52]. Beyond the bend, Ita \& Matsunaga (2011) [52] obtained the period-luminosity relation as

$$
M_{K_{\mathrm{s}}}=(-6.850 \pm 0.901) \log P+28.225 \pm 2.493-\mu_{0, \mathrm{LMC}}
$$

where $P(>400$ days $)$ is the pulsation period in days and $\mu_{0, \mathrm{LMC}}$ is the distance modulus toward LMC. Adopting $\mu_{0, \mathrm{LMC}}=18.493 \pm 0.048$ [87] and substituting $P=762.9$ days into Equation (3.10), Hachisu \& Kato (2018b) [35] obtained the absolute $K_{\mathrm{s}}$ magnitude of $M_{K_{\mathrm{s}}}=-10.01$. The average $K$ mag of V407 Cyg is $m_{K}=3.3$, and thus we have

$$
(m-M)_{K}=0.353 \times E(B-V)+5 \log (d / 1 \mathrm{kpc})+10=13.31,
$$

where we adopt the reddening law of $A_{K}=0.353 \times E(B-V)$ [7]. Substituting $E(B-V)=1.0$ into Equation (3.11), Hachisu \& Kato (2018b) [35] obtained the distance of $d=3.9 \mathrm{kpc}$. The distance modulus in $V$ band is calculated to be $(m-M)_{V}=16.1$ from Equation (3.1). 


\subsubsection{CSM-shock interaction}

Figure 14 shows a comparison of light curves of V407 Cyg and RS Oph on a logarithmic timescale. We stretch the timescale of RS Oph by a factor of $f_{\mathrm{s}}=4.5$ and shift down both the $V$ and SMEI light curves of RS Oph by 4.9 mag. The $V$ light curve of RS Oph (open blue squares) overlaps that of V407 Cyg (filled red circles) in the later phase, whereas the SMEI light curve of RS Oph (blue dots) overlap the $V$ light curve of V407 Cyg (filled red circles). The $V$ light curve of V407 Cyg decays as $F_{V} \propto t^{\alpha}$ ( $\alpha=-1.0$, denoted by the solid red line) in the early phase (up to $t \sim 45$ days).

Cool winds from the Mira-like companion form CSM around the binary (e.g., [66]). The nova ejecta collide with the CSM and form a strong shock (e.g, [83, 86]). Shore et al. (2011) [98] and Iijima (2015) [51] showed that the strong shock between the ejecta and CSM contributes to the emission lines and soft X-ray flux. Such an interaction between ejecta and CSM was frequently observed in supernovae Type IIn. For example, Moriya et al. (2013) [68] showed that $F_{v} \propto t^{\alpha}$ $(\alpha \approx-1.0)$ in the $V$-band for SN 2005ip. Thus, we interpret this early decay of $F_{V} \propto t^{-1}$ as the shock interaction. The $I_{\mathrm{C}}$ light curve of V407 Cyg also show a decline trend of $F_{V} \propto t^{-1.0}$, which is shifted down by 2.7 mag.

Considering the shock interaction, we summarize the evolution of V407 Cyg $V$ light curve as follows: in the early stage, just after the optical maximum, the ejecta collide with the CSM and produce a strong shock. This shock-heating contributes significantly to the $V$ brightness. Then, the shock broke out of the CSM approximately 45 days after the outburst (JD 2455310). Soon after the shock breakout, the $V$ light curve decays as $F_{v} \propto t^{-1.55}$, as shown in Figure 14. This $\alpha=-1.55$ is close to the universal decline law of $\alpha=-1.75$ (see [25]).

RS Oph decays as $F_{V} \propto t^{-1.55}$ in the $V$ band. The CSM shock is much weaker in RS Oph than in V407 Cyg, such that the $V$ light curve of RS Oph is close to that of the universal decline law $\left(F_{V} \propto t^{-1.75}\right)$ showing no indication of strong shock interaction $\left(F_{V} \propto t^{-1.0}\right)$. In other words, the shock interaction is strong enough to increase the continuum flux to $F_{v} \propto t^{-1.0}$ in V407 Cyg, but not enough in RS Oph. In contrast, the SMEI light curve of RS Oph almost obeys $L_{\mathrm{SMEI}} \propto t^{-1.0}$ like the early decline trend of V407 Cyg, where $L_{\text {SMEI }}$ is the SMEI band luminosity. This is because the SMEI magnitude is a wide-band (peak quantum efficiency at $700 \mathrm{~nm}$ with an FWHM of 300 $\mathrm{nm}$ ) and envelopes a very strong $\mathrm{H} \alpha$ line, which mainly comes from the shock interaction.

It should be noted that the three rapid-decline novae, V745 Sco, T CrB, and V1534 Sco, also have a RG companion but do not show clear evidence of shock-heating in their $V$ (or visual) light curves, because no part shows $F_{v} \propto t^{-1}$ (see Figure 16 and also Figure 2 of [35]). Their $V$ light curves almost overlap to that of V838 Her, which has a MS companion [35]. The X-ray fluxes of V407 Cyg, RS Oph, V745 Sco, and V1534 Sco were observed with Swift. Their origin could be shock-heating in the very early phase, before the SSS phase started. Munari et al. (2018) [74] showed no evidence of deceleration of ejecta in V1534 Sco. These indicate that the shape of $V$ light curve changes from that of V407 Cyg to RS Oph, and finally to V1534 Sco, depending on the strength of shock interaction. V407 Cyg shows a strongest limit of shock interaction while V1534 Sco corresponds to a weakest limit of shock [35]. 


\subsubsection{Fate of V407 Cyg}

Using the linear relation between $M_{\mathrm{WD}}$ and $\log f_{\mathrm{s}}$ in Figure 15, Hachisu \& Kato (2018b) [35] obtained the WD mass of $M_{\mathrm{WD}}=1.22 \pm 0.02 M_{\odot}$ for V407 Cyg. Here, they used the linear relation between $\log f_{\mathrm{s}}=0.3$ and $\log f_{\mathrm{s}}=1.1$ (right straight solid red line). Even if we assume the WD mass increases at the rate of $\dot{M}_{\mathrm{WD}}=1 \times 10^{-7} M_{\odot} \mathrm{yr}^{-1}$ [58], it takes $t_{\mathrm{Ia}}=(1.38-1.22) M_{\odot} / 1 \times$ $10^{-7} M_{\odot} \mathrm{yr}^{-1}=1.6 \times 10^{6} \mathrm{yr}$ to explode as a SN Ia. We do not expect that this high mass-accretion rate will continue for such a long time, because the Mira-like companion has already reached a very late evolution stage of an AGB star as suggested by the $\mathrm{SiO}$ maser observations [11, 9]. Therefore, Hachisu \& Kato (2018b) [35] concluded that V407 Cyg is not a progenitor of SNe Ia.

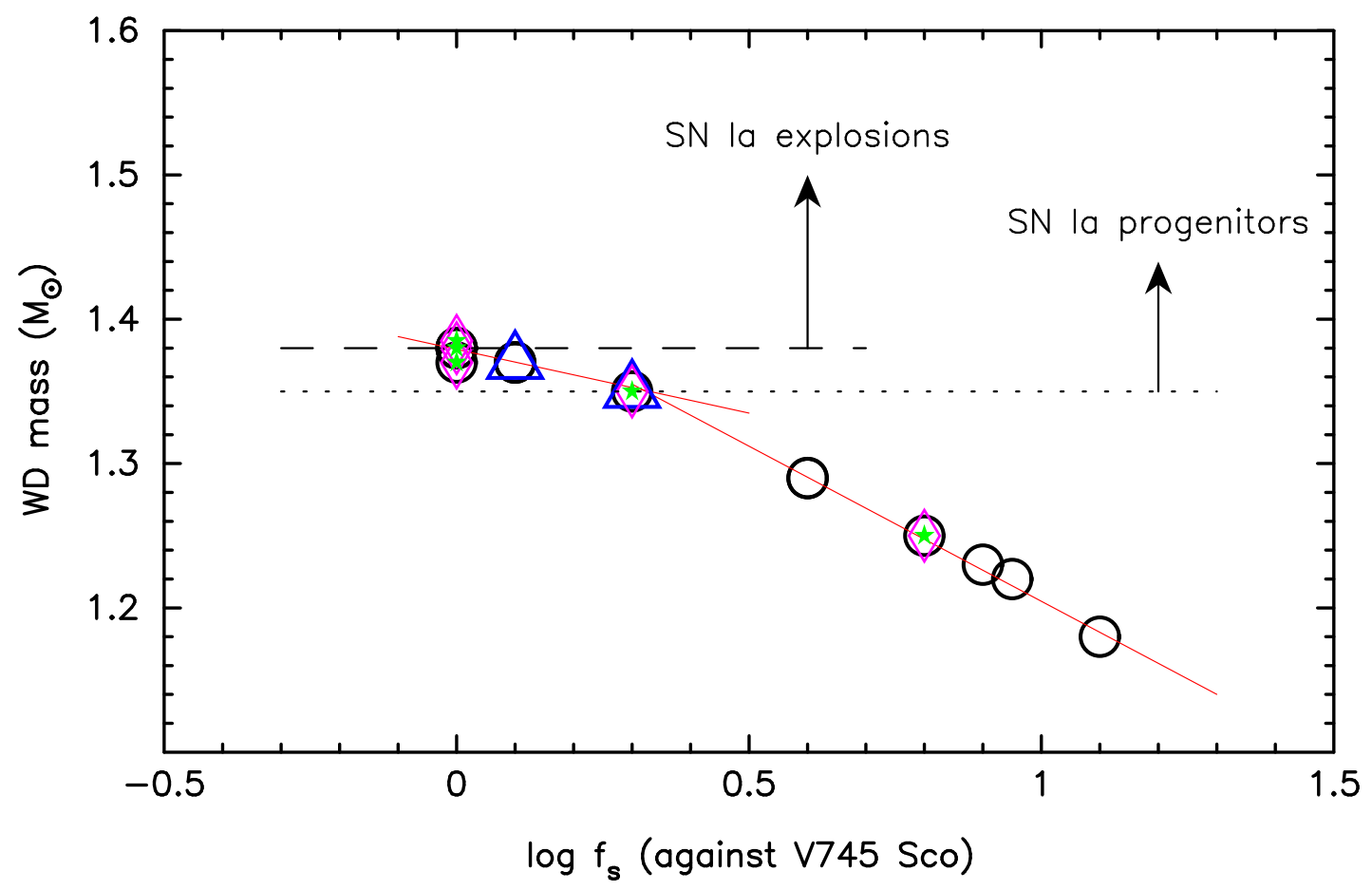

Figure 15: WD mass vs. timescaling factor $\log f_{\mathrm{s}}$ for selected recurrent and very fast novae. The timescaling factor is measured against that of V745 Sco $\left(f_{\mathrm{s}}=1.0\right.$ for V745 Sco). The data are taken from Table 3 of [35] and essentially the same as Figure 17 of [35].

\subsection{Light Curve Shapes of Recurrent and Fast Novae}

Hachisu \& Kato (2006) [25] found that the optical and NIR light curves of several novae follow a similar decline law. Moreover, they found that the time-normalized light curves are independent of the WD mass, chemical composition of the ejecta, and wavelength. They called this property the universal decline law. The universal decline law was examined first in several well-observed novae and later in many other novae ( 230 novae) [26, 27, 28, 31, 32, 33, 37, 38, 39, 56, 55]. Hachisu \& Kato (2006) [25] defined a unique timescaling factor of $f_{\mathrm{s}}$ for optical, UV, and supersoft X-ray light curves of a nova. The shortest timescales (smallest $f_{\mathrm{s}}$ ) correspond to the WD masses in the range $1.37-1.38 M_{\odot}$. Therefore, the shortest $f_{\mathrm{s}}$ systems are candidates for immediate progenitors of SNe Ia (e.g., [21, 40, 41, 42, 28, 32, 33]) as can be seen in Figure 15. 


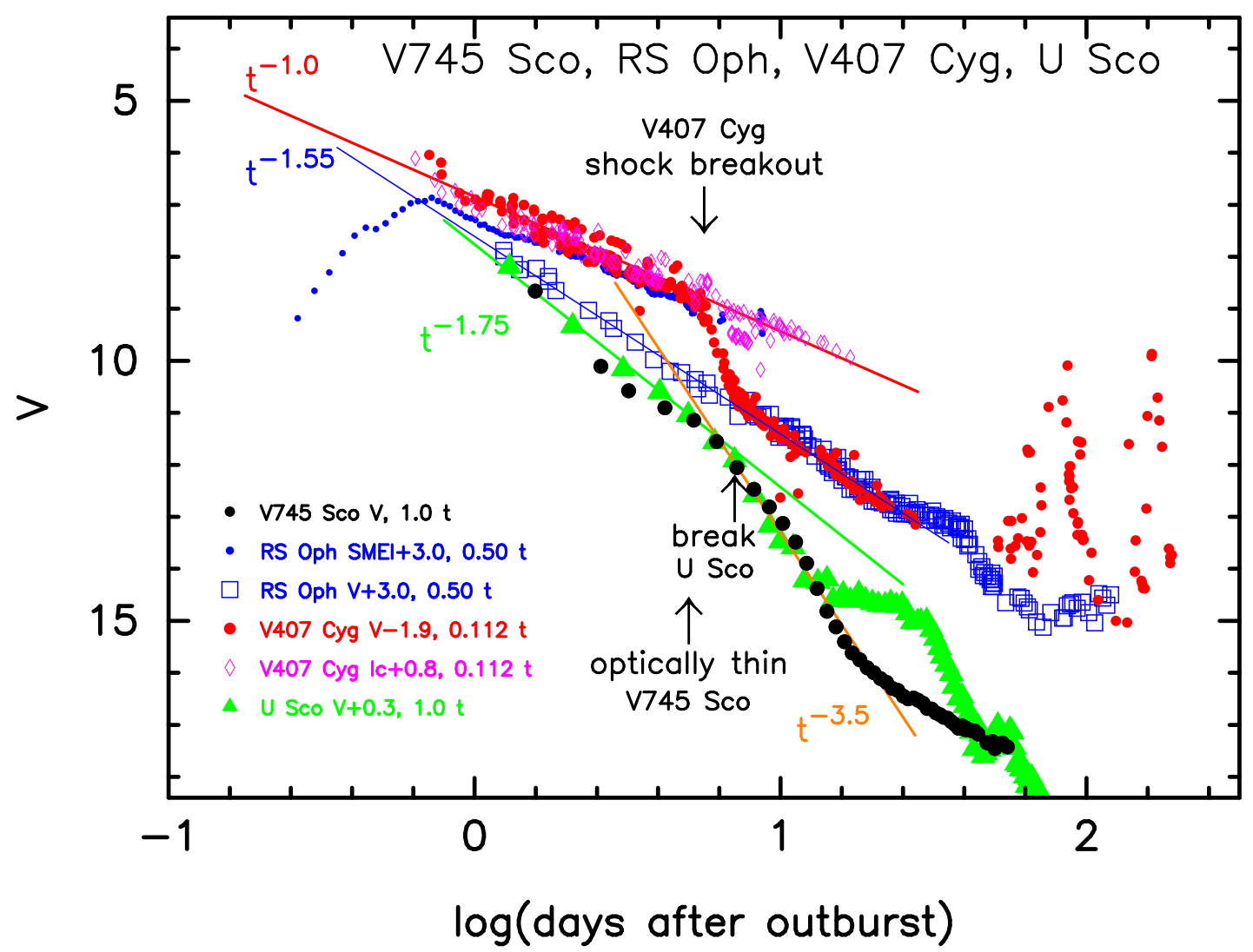

Figure 16: Three $V$ light curves of the V745 Sco (rapid decline), RS Oph (CSM shock), and U Sco (normal decline). We add the $V$ and $I_{\mathrm{C}}$ light curves of V407 Cyg (CSM shock) and the Solar Mass Ejection Imager (SMEI) magnitudes [48] of RS Oph for comparison. Each light curve is horizontally moved by $\Delta \log t=$ $\log f_{\mathrm{s}}$ and vertically shifted by $\Delta V$ with respect to that of $\mathrm{V} 745 \mathrm{Sco}$, as indicated in the figure by, for example, "RS Oph $\mathrm{V}+3.0,0.50 \mathrm{t}$," where $\Delta V=3.0$ and $f_{\mathrm{s}}=0.50$. The data are the same as those in Figure 1 of Hachisu $\&$ Kato (2018b). The filled black circles represent the $V$ light curve of the V745 Sco (2014) outburst. The filled red circles and open magenta diamonds denote the $V$ and $I_{\mathrm{C}}$ magnitudes of V407 Cyg, respectively. The blue dots and open blue squares denote the SMEI and $V$ magnitudes of the RS Oph (2006) outburst, respectively. The filled green triangles show the $V$ magnitudes of the U Sco (2010) outburst. The solid red line represents the decline trend of $F_{V} \propto t^{-1.0}$, solid blue line $F_{V} \propto t^{-1.55}$, solid green line $F_{V} \propto t^{-1.75}$, and solid orange line $F_{V} \propto t^{-3.5}$, where $t$ is the time from the outburst and $F_{V}$ is the flux at the frequency $v$.

There are exceptions of fast novae that do not follow the universal decline law. For example, as already shown in Figure 14, V407 Cyg shows a decay trend of $F_{V} \propto t^{-1.0}$ in the early $V$ light curve, whereas the universal decline law of novae follows $F_{V} \propto t^{-1.75}$, as in the early decay phase of U Sco as shown in Figure 16. V745 Sco is a recurrent nova and does not show a substantial slope of $F_{V} \propto t^{-1.75}$ in the early decay phase, but rather a steeper decline of $F_{V} \propto t^{-3.5}$, as shown in Figure 16. We have to clarify the reasons for these exceptions and determine the WD mass based on our model light curve fitting.

The slow decline of $F_{v} \propto t^{-1}$ is owing to the contribution of shock heating between ejecta and CSM as discussed in the case of V407 Cyg. On the other hand, the fastest group, V745 Sco, T CrB, V838 Her, and V1534 Sco have no or too short duration of the universal trend of $F_{v} \propto t^{-1.75}$ 
(or $\propto t^{-1.55}$ ) and decline like $F_{v} \propto t^{-3.5}$, much faster than the universal trend. This is because their initial hydrogen-rich envelope masses are too small to follow the universal trend (see Figure 1 and discussion in [35]). Hachisu \& Kato (2018b) [35] classified the four novae, V745 Sco, T CrB, V838 Her, and V1534 Sco, as the V745 Sco type (rapid decline), the two novae, RS Oph and V407 Cyg, as the RS Oph type (CSM shock), and the two novae, U Sco and CI Aql, as the U Sco type (normal decline), depending on their light curve shape types (see Figure 16). The $V$ light curves of these novae almost overlap each other in the same group, if we properly stretch or squeeze in the time-direction (timescaling law).

\section{Concluding Remarks}

We summarize the evolution of a nova outburst (see Figure 4 for a cartoon of nova outbursts). During the outburst, the WD envelope expands to engulf the companion star. After the maximum expansion of pseud-photosphere, a large part of the envelope matter is blown in the wind and the photosphere begins to shrink and moves further inside. The companion eventually emerges from the WD photosphere and an accretion disk may appear or reestablished again. The photosphere further shrinks and the optically thick wind stops. Hydrogen nuclear burning stops and the nova enters a cooling phase. Hard X-rays (and/or GeV gamma-rays) may originate from internal shocks between ejecta (or a bow shock between ejecta and the companion) from stage (a) to (f) as indicated by a dashed line as shown in Figure 4. The optical flux dominates the spectra from stage (a) to (b). The ultraviolet (UV) flux dominates from stage (b) to (f). Then the supersoft X-ray flux replaces the UV flux from stage (f) to (g).

The main emitting wavelength region of a nova moves first from supersoft X-ray (X-ray flash), to UV (UV flash), and to optical (at maximum), as the photosphere expands (see Figure 3 for a cartoon). After the optical maximum, the pseud-photosphere begins to shrink and an optically thin region develops. Free-free emission dominates the spectrum and the optical and NIR brightnesses decay along the universal decline law of $F_{V} \propto t^{-1.75}$ ( or $t^{-1.55}$ ). The wind mass-loss rate sharply drops and finally vanishes. In this phase, the slope of free-free emission decays like $F_{v} \propto t^{-3.5}$. The shock interaction between ejecta and circumstellar matter slows down the decay of optical flux as $F_{V} \propto t^{-1.0}$ in the early phase of V407 Cyg. The timescale of a nova outburst depends mainly on the WD mass, so that we are able to estimate the WD mass by fitting model light curves with multiwavelength observation (see Figure 15 for the WD mass vs. timescale relation).

\section{Acknowledgments}

This research has been supported in part by the Grants-in-Aid for Scientific Research (15K05026, 16K05289) of the Japan Society for the Promotion of Science.

\section{References}

[1] Abdo, A. A., Ackermann, M., Ajello, M., et al. 2010, Science, 329, 817

[2] Ackermann, M., Ajello, M., Albert, A., et al. 2014, Science, 345, 554 
[3] Andreae, J., Drechsel, H., Snijders, M. A. J., \& Cassatella, A. 1991, A\&A, 244, 111

[4] Arenas, J., Catalán, M. S., Augusteijn, T., \& Retter, A. 2000, MNRAS, 311, 135

[5] Barry, R. K., Mukai, K., Sokoloski, J. L., et al. 2008, RS Ophiuchi (2006) and the Recurrent Nova Phenomenon, ASP Conference Series, 401, eds. A. Evans, M. F. Bode, T. J. O’Brien, \& M. J. Darnley, San Francisco, Astronomical Society of the Pacific, 52

[6] Brandi, E., Quiroga, C., Mikołajewska, J., Ferrer, O. E., \& García, L. G. 2009, A\&A, 497, 815

[7] Cardelli, J. A., Clayton, G. C., \& Mathis, J. S. 1989, ApJ, 345, 245

[8] Cassatella, A., Altamore, A., \& González-Riestra, R. 2002, A\&A, 384, 1023

[9] Cho, S.-H., Kim, J., \& Yun, Y. 2015, J. Korean Astr. Society, 48, 267

[10] Connelley, M., \& Sandage, A. 1970, PASP, 70, 600

[11] Deguchi, S., Koike, K., Kuno, N., et al. 2011, PASJ, 63, 309

[12] Denissenkov, P. A., Herwig, F., Bildsten, L., \& Paxton, B. 2013, ApJ, 762, 8

[13] Doherty, C. L., Gil-Pons, P., Siess, L., Lattanzio, J. C., \& Lau, H. H. B. 2015, MNRAS, 446, 2599

[14] Downes, R. A., \& Duerbeck, H. W. 2000, AJ, 120, 2007

[15] Duerbeck, H. W., Geffert, M., Nelles, B., Dummler, R., \& Nolte, M. 1984, IBVS, 2641, 1

[16] Esipov, V. F., Kolotilov, E. A., Shenavrin, V. I., et al. 2015, Baltic Astronomy, 24, 353

[17] Evans, P. A., Beardmore, A. P., Page, K. L., et al. 2009, MNRAS, 397, 1177

[18] Friedjung, M. 1987, A\&A, 180, 155

[19] Gehrz, R. D., Harrison, T. E., Ney, E. P., et al. 1988, ApJ, 329, 894

[20] Gehrz, R. D., Truran, J. W., Williams, R. E., \& Starrfield, S. 1998, PASP, 110, 3

[21] Hachisu, I., \& Kato, M. 2001, ApJ, 558, 323

[22] Hachisu, I., \& Kato, M., 2003a, ApJ, 590, 445

[23] Hachisu, I., \& Kato, M., 2003b, ApJ, 598, 527

[24] Hachisu, I., \& Kato, M., 2004, ApJL, 612, L57

[25] Hachisu, I., \& Kato, M. 2006, ApJS, 167, 59

[26] Hachisu, I., \& Kato, M. 2007, ApJ, 662, 552

[27] Hachisu, I., \& Kato, M. 2009, ApJL, 694, L103

[28] Hachisu, I., \& Kato, M. 2010, ApJ, 709, 680

[29] Hachisu, I., \& Kato, M. 2012, Baltic Astronomy, 21, 68

[30] Hachisu, I., \& Kato, M. 2014, ApJ, 785, 97

[31] Hachisu, I., \& Kato, M. 2015, ApJ, 798, 76

[32] Hachisu, I., \& Kato, M. 2016a, ApJ, 816, 26

[33] Hachisu, I., \& Kato, M. 2016b, ApJS, 223, 21

[34] Hachisu, I., \& Kato, M. 2018a, ApJ, 858, 108 
[35] Hachisu, I., \& Kato, M. 2018b, ApJS, in press (arXiv:1805.09932)

[36] Hachisu, I., Kato, M., Kato, T., \& Matsumoto, K. 2000, ApJL, 528, L97

[37] Hachisu, I., Kato, M., Kiyota, S., et al. 2006, ApJL, 651, L141

[38] Hachisu, I., Kato, M., \& Luna, G. J. M. 2007, ApJL, 659, L153

[39] Hachisu, I., Kato, M., \& Cassatella, A. 2008, ApJ, 687, 1236

[40] Hachisu, I., Kato, M., \& Nomoto, K. 1996, ApJ, 470, L97

[41] Hachisu, I., Kato, M., \& Nomoto, K. 1999a, ApJ, 522, 487

[42] Hachisu, I., Kato, M., Nomoto, K., \& Umeda, H. 1999b, ApJ, 519, 314

[43] Harrison, T. E., Johnson, J. J., \& Spyromilio, J. 1993, AJ, 105, 320

[44] Henze, M., Darnley, M. J., Williams, S. C., et al. 2018, ApJ, 857, 68

[45] Henze, M., Ness, J.-U., Darnley, M., et al. 2015, A\&A, 580, 46

[46] Hjellming, R. M., van Gorkom, J. H., Seaquist, E. R., et al. ApJL, 305, L71

[47] Hoard, D. W., Wachter, S., Clark, L. Lee, \& Bowers, T. P. 2002, ApJ, 565, 511

[48] Hounsell, R., Bode, M. F., Hick, P. P., et al. 2010, ApJ, 724, 480

[49] Iben, I. Jr. 1982, ApJ, 259, 244

[50] Iijima, T. 2009, A\&A, 505, 287

[51] Iijima, T. 2015, AJ, 150, 20

[52] Ita, Y., \& Matsunaga, N. 2011, MNRAS, 412, 2345

[53] Kantharia, N. G., Dutta, P., \& Roy, N. 2016, MNRAS, 456, L49

[54] Kato, M., \& Hachisu, I. 1994, ApJ, 437, 802

[55] Kato, M., \& Hachisu, I. 2012, Bull. Astr. Soc. India, 40, 393

[56] Kato, M., Hachisu, I., \& Cassatella, A. 2009, ApJ, 704, 1676

[57] Kato, M., Saio, H., Hachisu, I., \& Nomoto, K. 2014, ApJ, 793, 136

[58] Kato, M., Saio, H., \& Hachisu, I. 2017a, ApJ, 838, 153

[59] Kato, M., Saio, H., \& Hachisu, I. 2017b, ApJ, 844,143

[60] Kato, M., Saio, H., Henze, M. et al. 2016, ApJ, 830, 40

[61] Kolotilov, E. A., Shenavrin, V. I., Shugarov, S. Yu., \& Yudin, B. F. 2003, Astronomy Reports, 47, 777

[62] Kovetz, A. 1998, ApJ, 495, 401

[63] Li, X.-D., \& van den Heuvel, E. P. J., 1997, A\&A, 322, L9

[64] Marshall, D. J., Robin, A. C., Reylé, C., Schultheis, M., \& Picaud, S. 2006, A\&A, 453, 635

[65] Mikołajewska, J., \& Shara, M. M. 2017, ApJ, 847, 99

[66] Mohamed, S., \& Podsiadlowski, Ph. 2012, Baltic Astronomy, 21, 88

[67] Morii, M., Yamaoka, H., Mihara,T., Matsuoka, M., \& Kawai, N. 2016, PASJ, 68, S11

[68] Moriya, T. J., Maeda, K., Taddia, F., et al. 2013, MNRAS, 435, 1520 
[69] Mróz, P., Poleski, R., Udalski, A., et al. 2014, MNRAS, 443, 784

[70] Morris, P. J., Cotter, G., Brown, A. M., \& Chadwick, P. M. 2017, MNRAS, 465, 1218

[71] Mukai, K., \& Ishida, M. 2001, ApJ, 551, 1024

[72] Munari, U., Margoni, R., \& Stagni, R. 1990, MNRAS, 242, 653

[73] Munari, U., Joshi, V. H., Ashok, N. M., et al. 2011b, MNRAS, 410, L52

[74] Munari, U., \& Banerjee, D. P. K. 2018, MNRAS, 475, 508

[75] Nariai, K., Nomoto, K., \& Sugimoto, D. 1980, PASJ, 32, 473

[76] Nelson, T., Donato, D., Mukai, K., Sokoloski, J., \& Chomiuk, L. 2012, ApJ, 748, 43,

[77] Nishimaki, Y., Yamamuro, T., Motohara, K., Miyata, T., \& Tanaka, M. 2008, PASJ, 60, 191

[78] Nomoto, K. 1982, ApJ, 253, 798

[79] Nomoto, K., Saio, H., Kato, M., Hachisu, I., 2007, ApJ, 663, 1269

[80] Öegelman, H., Krautter, J., \& Beuermann, K. 1987, A\&A, 177, 110

[81] O’Brien, T. J., Bode, M. F., Porcas, R. W., et al. 2006, Nature, 442, 279

[82] Orio, M., Rana, V., Page, K. L., Sokoloski, J., Harrison, F. 2015, MNRAS, 448, L35

[83] Orlando, S., \& Drake, J. J. 2012, MNRAS, 419, 2329

[84] Page, K. L., Osborne, J. P., Kuin, N. P. M., et al. 2015, MNRAS, 454, 3108

[85] Pagnotta, A., Schaefer, B. E., Clem, J. L., et al. 2015, ApJ, 811, 32

[86] Pan, K.-C., Ricker, P. M., \& Taam, R. E. 2015, ApJ, 806, 27

[87] Pietrzyński, G., Graczyk, D., Gieren, W., et al. 2011, Nature, 495, 76

[88] Prialnik, D. 1986, ApJ, 310, 222

[89] Prialnik, D., \& Kovetz, A. 1995, ApJ, 445, 789

[90] Robb, R. M., \& Scarfe, C. D. 1995, MNRAS, 273, 347

[91] Saizar, P., Starrfield, S., Ferland, G. J., et al. 1991, ApJ, 367, 310

[92] Schaefer, B. E. 2009, ApJ, 697, 721

[93] Schaefer, B. E. 2010, ApJS, 187, 275

[94] Schaefer, B. E., Pagnotta, A., LaCluyze, A. P., et al. 2011, ApJ, 742, 113

[95] Schlafly, E. F., \& Finkbeiner, D. P. 2011, ApJ, 737, 103

[96] Seaton, M. J. 1979, MNRAS, 187, 73P

[97] Sekiguchi, K., Whitelock, P. A., Feast, M. W., et al. 1990, MNRAS, 246, 78

[98] Shore, S. N., Wahlgren, G. M., Augusteijn, T., et al. 2011, A\&A, 527, A98

[99] Siviero, A., \& Munari, U. 2006, CBET, 502, 1

[100] Snijders, M. A. J. 1987, ApSS, 130, 243

[101] Sokoloski, J. L., Luna, G. J. M., Mukai, K., \& Kenyon, S. J. 2006, Nature, 442, 276

[102] Starrfield, S., Truran, J. W.., Sparks, W. M., \& Kutter, G. S. 1972, ApJ, 176, 169 
[103] Thoroughgood, T. D., Dhillon, V. S., Littlefair, S. P., Marsh, T. R., \& Smith, D. A. 2001, MNRAS, 327,1323

[104] van den Bergh, S., \& Younger, P. F. 1987, A\&AS, 70, 125

[105] van den Heuvel, E. P. J., Bhattacharya, D., Nomoto, K., \& Rappaport, S. A. 1992, A\&A, 262, 97

[106] Whitelock, P. A., Feast, M. W., \& van Leeuwen, F. 2008, MNRAS, 386, 313

[107] Wolf, W. M., Bildsten, L., Brooks, J., \& Paxton, B. 2013, ApJ, 777, 136

[108] Woodward, C. E., Gehrz, R. D., Jones, T. J., Lawrence, G. F., \& Skrutskie, M. F. 1997, ApJ, 477, 817

[109] Wright, A. E., \& Barlow, M. J. 1975, MNRAS, 70, 41

\section{DISCUSSION}

Ryoko Ishioka: Are there any cases that WD mass is measured by any other ways such as RV or eclipses?

Hachisu: For example, the dynamical mass of V603 Aql was estimated by Arenas et al. (2000, MNRAS, 311, 135) [4] to be $M_{\mathrm{WD}}=1.2 \pm 0.2 M_{\odot}$. This mass is consistent with our result of $M_{\mathrm{WD}}=1.2 M_{\odot}$ with multifrequency light curve fitting (Hachisu \& Kato, 2014, ApJ, 785, 97) [30].

Sölen Balmann: How do you explain or rather find the M31 nova (Martin Henze nova) to appear and show different recurrent timescale?

Hachisu: One possible explanation is variation in the mass accretion rate during the last (2016 outburst) and the previous (2015 outburst). A lower mass-accretion rate makes a longer duration of inter-pulse. (Note added in proof: Henze et al. (2018, ApJ, 857, 68) [44] discussed the longer recurrence period based on the possible lower mass accretion rate.) 